\title{
ANALOGIES OF GENETIC AND CHEMICAL CODE
}

\author{
Miloje M. Rakočević \\ Department of Chemistry, Faculty of Science, University of Niš, Serbia \\ (E-mail: mirkovmile@mts.rs; mirkovmiloje@gmail.com; www.rakocevcode.rs)
}

\begin{abstract}
Starting from the previously established hypothesis on the existence of "the coherence of the chemical and genetic code" (Rakočević, 1991), new facts and new insights on the existence of essential analogies between the genetic and chemical code are presented, whereby the chemical code is derived from the original Mendeleev's work on the periodic system of chemical elements. When, namely, the 14 lanthanides are scheduled into 14 groups of Periodic system of chemical elements (PSE) (Rakočević, 1991), in accordance to Mendeleev's original manuscript tables, then are revealed some very specific mathematical and chemical regularities within the PSE; among others, PSE appears to be a code - the chemical code - in an entity analogy with the terrestrial genetic code. These regularities correspond to the mathematical regularities within the genetic code. Between others relations, it appears also a correspondance between the distribution of codons in the Genetic Code Table and the distribution of chemical elements in the PSE with respect to their even/odd parity and stability/instability of the isotopes. Based on the significant mathematical expressions it is showed a new essence of coding formalism in natural code as such: it cannot be reduced only to two alphabets (which are consequences, and not the cause); a natural code is such an essence that can be represented by an appropriate mathematical expression, which contains a sequence from the series of natural numbers within itself. From this follows that both are natural codes, the Genetic code as well as the Periodic system of chemical elements (PSE).
\end{abstract}

Keywords. Genetic code; periodic system of chemical elements; chemical code; lanthanides; protein amino acids; stereochemical types of amino acids; diversity types of amino acids; molecular diversity; golden mean; generalized golden mean.

\section{Preliminaries}

\subsection{Analogies with quantum physics}

V. Shcherbak showed that a specific classification of the protein amino acids (AAs), "canonical" amino acids within the genetic code (GC), reveals some arithmetical regularities and stated that "the physical nature of such a phenomenon is so far not clear" (Shcherbak, 1993). There is a similar statement of A. Verkhovod in his work on the same subject and published a few months later: "The nature of these mechanisms is presently unknown" (Verkhovod, 1994). In the second part of his rsearches Shcherbak (1994) "seeks to identify a trend of possible physical interpretation of the new code properties", and reveals that in the question are "analogies with quantum physics." By this, the Shcherbak's classification refers to the splitting into four-codon and non-four-codon AAs, based on the original work of Rumer (Rumer, 1966) (Table A. 1 in Appendix A). If so, then there are analogies in the next sense: 01 x 111 of electrons in the half-filled state of the atom orbitals, or $01 \times 222$ in the full-filled state, according to Hund's rule, versus $10 \times 111$ nucleons in 15 side chains as well as in 15 "heads", i.e. amino acid functional groups (after Shcherbak: "standard boxes") of 15 non-four-codon AAs, what equals 
10 x 222 in 15 whole molecules; at the same time there are $01 \times 333$ nucleons in 8 side chains of 8 four-codon AAs (for details see: Shcherbak, 1994). ${ }^{1}$

Considering that $01 \times 333$ can be "read" as $01 \times \underline{\mathbf{3}}^{\wedge} 2 \mathrm{PQ}$, then we find that in 8 "heads" of 8 four-codon AAs there are $01 \times \underline{\mathbf{4}}^{\wedge} 2 \mathrm{PQ}$, or in total $01 \times \underline{\mathbf{5}}^{\wedge} 2 \mathrm{PQ}$ of nucleons, what is again an analogy with quantum physics, as well as with two Mendeleev principles at the same time (see Remarks 1-3).

Remark 1. Whether it is a move for one electron in filling of the orbital, or for a single numbering unit in filling the positions in the number record of the number of nucleons, both have correspondence with two key Mendelevian principles: with the principle of continuity and the principle of minimum change. In addition to this, it should be noted that this is a special case of a minimum change - a change for a unit $;^{2}$ and there is also an analogy with the changes on the Gray code model of the genetic code, ${ }^{3}$ as well as on the GC binary tree that follows from the Gray Code. ${ }^{4}$

Remark 2. The uniqueness of the number 037 in the Shcherbak's pattern "Prime Quantum 037" (PQ) follows from the uniqueness of a specific sequence, generated from the series of natural numbers: $\left(13_{4}\right.$, $\left.25_{7}, 37_{10}, 49_{13}, \ldots\right)^{5}$, for which two Mendeleev's principles also are valid. [The validity through the sequence 1, 2, 3, 4, etc., in front position, and through the sequence 3, 5, 7, 9, etc., in ending position.]

Remark 3. The chemical and formal bond between four-codon and non-four-codon amino acids is described in the next Section. However, one specific formal-mathematical relation, in a certain way hidden, ${ }^{6}$ we present here: $\left(\underline{1} 0 \times 3^{\wedge} \underline{1} \mathrm{PQ}\right)$ nucleons within 15 side chains of 15 non-four-codon AAs versus $\left(0 \underline{1} \times 3^{\wedge} \underline{2} \mathrm{PQ}\right)$ nucleons within 8 side chains of 8 four-codon AAs. It should be noted here that the "15" encoded entities are actually 16 encoded entities because the $16^{\text {th }}$ entity joins a "stop" signal. In this case, the ratio of four-codon and non-four-codon situations is in a strictly symmetrical manner of view, 8 : $16=1: 2$ (cf. Survey 1 and Table A.1).

As far as Verkhovod's work is concerned, he showed that the number of nucleons in the 23 amino acid molecules in the standard GCT directly corresponds with the sequence of the series of natural numbers (in decimal numbering system) in a specific way, through the "game" of the visible and invisible image in the "mirror": 456/789 and 987/654. Namely, in amino acid side chains, within $\left(1^{\text {th }}+4^{\text {th }}\right)$ rows, as well as within $\left(2^{\text {nd }}+3^{\text {rd }}\right)$ columns there are 654 nucleons in both

\footnotetext{
${ }^{1}$ Shcherbak (1994, p. 475): "The cause of the regularity lies in the properties of three-digit number notations, multiples of 37 in the decimal additive-position system. The notations of the sums, the nucleon number of the amino acid standard box 074 and Prime Quantum 037 ... can be seen as a fragmentary pattern of the general regularity of Table 1" (here: Table A.2).

2 "Such a strict regularity in a change ... exactly for a unit shall be specified (and defined) as the unit change law" (Rakočevič, 1994, p. 36).

3 "... any two adjacent symbols [in Gray code] differ at only one bit" (Swanson, 1984, p. 188).

4 "The binary-code tree [of the GC] corresponds per se with the Gray code model of the genetic code (Fig. 1 in Swanson, 1984 p. 188)" (Rakočević, 1998, p. 283).

${ }^{5}$ Is it just as a curiosity, or more than that, this can not be known, but the fact is that the number of verses in the 100 songs of Divine Comedy of Dante Aligierie corresponds with this sequence: the number of verses in any of the 100 songs is so large that the sum of the digits of the numeral record is 4, 7,10 or 13, without any exception.

${ }^{6}$ We say "hidden relation," since from the aspect of valid paradigms in the current science it is not expected that "quantization" in positions in the decimal numbering records could in any way be analogous to quantization in quantum physics.
} 
area; vice versa, within $\left(1^{\text {th }}+4^{\text {th }}\right)$ columns, as well as within $\left(2^{\text {nd }}+3^{\text {rd }}\right)$ rows there are 789 nucleons in each of two area (Figure 2 in Verkhovod, 1994).

\subsection{Agreement-disagreement principle}

As we can see in above presented classification of AAs appears a specific unity of chemistry and mathematical formalism, which in this paper will be analyzed in details, starting from both works, Rumer's and Shcherbak's. In his first work on the genetic code, Rumer (1966) shows that four variations of nucleotide doublet $\mathrm{CG}(\mathrm{CC}, \mathrm{GG}, \mathrm{CG}, \mathrm{GC})$ with 6 hydrogen bonds (higher rank!) encode four-codon AAs (higher rank!), while four variations of UA (UU, AA, UA, AU), each with 4 hydrogen bonds (lower rank!) encode non-four-codon AAs (also lower rank!). However, the situation is more "complicated" in the coding with nucleotide doublets that have 5 hydrogen bonds each. There is a characteristic "crossing", but also in relation to a strict chemical distinction. Thus, nucleotide doublets that in the second position possess a more complex nucleotide (CA \& UG), i.e. the nucleotides of higher rank, encode non-four-codon AAs, what is per se a situation of lower rank. On the other hand, nucleotide doublets that in the second position possess a less complex nucleotide (AC \& GU), i.e. the nucleotides of lower rank, encode four-codon AAs, what is the situation of higher rank. Finally, pyrimidine doublets (UC \& CU) (lower rank!) encode four-codon AAs (higher rank!), while purine doubles (GA \& AG) (higher rank!) encode non- four-codon AAs (lower rank!).

As we see, the state of things is such that the disagreement in the number of hydrogen bonds $(6 \& 4)$ is accompanied by an agreement in the rank of the complexity of the molecules and / or the complexity of the coding process; vice versa, the agreement in the number of hydrogen bonds ( $5 \& 5)$ is accompanied by disagreement in the ranking. In a certain way, here we indeed have an analogy with quantum physics, more precisely with Heisenberg's uncertainty principle (in the form of an agreement/disagreement principle).

\subsection{Some other mathematical formalisms}

Independently of Shchrbak and Verkhovod, other authors, during the last decades, have also presented other different arrangements in which chemical distinctions are accompanied by some kinds of mathematical formalism. So, R. Swanson has shown that the genetic code can be reduced into a strict mathematical-formal model, determined with the "Gray code binary symbols for numbers 0-63", and also reduced into the "Codon path cube" in which "all 64 codons are displayed", and where "the three edges of the cubes represent the three positions in a codon" (Swanson, 1984). By this, in both cases the principles of minimum change and continuity are valid, the same principles which are valid for the arrangement of chemical elements in the periodic system of D.I. Mendeleev.

R. Swanson, however, showed another more important thing. The "Codon ring" in the form of a Gray code model, which we have in GC (input!) and the "Mutation ring" of the amino acids, which is the result of the evolution of proteins in the terrestrial organisms (output!) are more than very similar - mutatis mutandis they are the same. With this insight, she rightly states that these findings "provides a new standpoint for addressing questions of selection vs random drift in the evolution of the code." The additional reason for this reexamination is the fact that in both rings (Codon and Mutation ring) AAs are classified into small/large and inner/outer, in terms of their 
position in proteins, with directly visible certainty/uncertainty of these positions, which is again an analogy with quantum physics, i.e. with Heisenberg's uncertainty principle.

Using the same rules ${ }^{7}$ that were applyed to the generation of the Gray Code model of GC, it was possible to obtain a six-bit binary tree of GC, and show that the GC is determined by the Golden mean (Rakočević, 1998b). On the other hand, if the splitting of the amino acids into two (by enzymes aminoacyl-tRNA synthetases handled) groups, instead in the standard Genetic Code Table (GCT) (Wetzel, 1995), is made in Codon path cube, the significant regularities are noted the separating of two classes of AAs with only one exception (Rakočević, 1997a) (see Table B.1 in Appendix B).

Remark 4. The six-bit binary tree is specific, in addition to everything else, in following: only at such a binary tree the sums of numbers within two inner branches (two octets) corresponds to the first pair of friendly numbers $(220+284=504)$; and each two adjacent branches give the same sum (504); all together, a kind of logical square is realized: (0) $220+284=504$; (1) $156+348=504$; (2) $92+412=$ $504 ;(3) 28+476=504$. On the other hand, the sum of the numbers of the first quartet is 6 , the first octet 28; the sum of numbers on the left half (which coincides with the first half of the GCT), from 0 to 31, is 496 , which in turn is the realization of the first three perfect numbers $(6,28,496)$. If, however, we count all numbers $0-63$, then go back (cyclic!), where 0 (zero) becomes to be 127 , then the sum of all numbers within the sequence $0-127$ equals 8128 , which is actually the fourth perfect number. After these insights, it is clearer the analogy between the binary records of 64 codons in the genetic code and 64 hexagrams in the Chinese book I Ching, at least three thousand years old; the records, on the six-bit binary-code tree in both cases (Stent, 1969; Schoenberger, 1951; Rakočević, 1994) (www.rakocevcode.rs).

A year later, after R. Swanson's published work, an article more about specific mathematical formalism, accompanied by the chemical distinctions of AAs according to the number of hydrogen atoms in their molecules has been published (Sukhodolets, 1985). Such a distinction corresponds with the characteristic sequence from a series of natural numbers: $5,(6), 7,8,9,10$, $11,12,13,14$ of hydrogen atoms (Appendix C).

On all the said and some other similar investigations of mathematical regularities of the GC one can see the following works: (Sukhodolets, 1985; Leunissen and De Jong, 1986; Koruga, 1992; Madox, 1992, 1994; Shcherbak, 1993-2008; Damjanović, 1998-2006; Qiu and Zhu, 2000; Yang, 2004; Dragovich, 2006-2012; Negadi, 2009-2014; Castro-Chavez, 2010, 2011; Mišić, 2011; Petoukhov, 2014; 2016; Wohlin, 2015; Rakočević, 1988-2014).

\section{New insights}

\subsection{The problem of lanthanides arrangement in the PSE}

The reason why it has not been previously noted the possible analogies between mathematics of the GC and mathematics of the PSE, lies in the fact that all 14 lanthanides are placed in the third group of PSE, i.e. at the same position where is the Lanthanum. However, this is in disagreement with Mendeleev's approach that every element in the PSE should have its own

\footnotetext{
7 "The most important characteristic of a codon is whether it has a purine (most significant bit 1) or a pyrimidine (most significant bit 0) at the middle base position ...", etc (Swanson, 1984, p. 188).
} 
position. Namely, the book of B.M. Kedrov (Kedrov, 1977, p. 188, Table 16) contains a variant of Mendeleev's Periodic Table, in which Mendeleev did not formally indicated the groups, but it is evident that each element occupies one position. In that Table, lanthanum is located in the third group, Cerium in the fourth group and so consequently all other 13 elements, although two elements (Pm \& Lu) were not known in Mendeleev's time. (Some Mendeleev's manuscript Tables can be seen in the author website, $w w w$.rakocevcode. $r s.)^{8}$

This problem with lanthanides positions in the PSE is still actual, because recently IUPAC (International Union of Pure and Applied Chemistry) has launched a new research project which should determine whether lanthanum with atomic number 57, or the last lanthanide, Lutetium with atomic number 71, should be written in the formal Table of PSE (see Appendix D).

Following Mendeleev's methodology, it was possible to show that the 14 lanthanides require exposition into 14 groups of the PSE. Then together with zeroth group, there are 15 groups (Rakočević, 1991) (see Table 1 in this paper). If we have such an arrangement, then it is easy to recognize not only arithmetical but also some algebraic regularities in the PSE. In a previous work (Rakočević, 1991) we proposed a hypothesis that the PSE of the short period groups corresponds to the Boolean cube as well as the PSE of the long period groups to the Boolean hypercube $;^{9}$ the role of the $16^{\text {th }}$ group in such a case (in a cyclic ordering) plays either zeroth group or the first one. In fact we mainly pay attention to this chemical code, because it is an analog of the genetic code. ${ }^{10}$ [Mendeleev also entered the elements of the first group - copper, silver and gold - twice, at the beginning and at the end of the PSE (Kedrov, 1977, p. 128, photocopy XII). $]^{11}$

\subsection{Specific arithmetical patterns}

Table 1 (in relation to Survey 2) shows that for $\left[(\mathrm{s} \& \mathrm{p}), \mathrm{d}\right.$, f] elements, ${ }^{12}$ to the stability/instability border in PSE (to the Po-84), we have 8 times the pattern 5-3-1; then 2 times the pattern 0-3-1 and 4 times the pattern 0-0-1. All together 9-4-1 elements: 9 elements 8 times; 4

\footnotetext{
${ }^{8}$ There was, however, an attempt to "integrate" the lanthanides into the Periodic System, by Charles Janet (18491932), so each of them was in a separate group. Unfortunately, it was only in our time recognized that Charles Janet was "unrecognized genius of the Periodic System" (Stewart, 2010).

9 "Such a surprisingly simple model at the same time represents the Logical-Informational and GeometricalHomeomorphic-Topological model (LIGHT) of the cube-hypercube with an inscribed sphere-hypersphere." (Rakočević, 1991, p. 1).

$10 " . .$. the chemical code, built on the very principles mentioned and in complete accordance with the genetic code. ... All the relations in the chemical code and the genetic code are in accordance with periodicity and cyclicity of the natural number system ..." (Rakočević, 1991, p. 1).

${ }^{11}$ In this paper, we will deal with only the standard genetic code, with the 20 canonical (protein) amino acids, and all other variants of the genetic codes will be considered as 'deviant codes' (Weaver, 2012, pp. 568-569). These codes, namely, do not change nothing on the fact, when it comes to the genetic code as "amino acid code" (Swanson, 1984), because they are represented in all variants with the same 20 protein amino acids. (The only exceptions are Pyrrolyisine and Selenocysteine, presented at some very few organisms.) [Knight at al., 2001, p. 49: "The genetic code evolved in two distinct phases. First, the 'canonical' code emerged before the last universal ancestor; subsequently, this code diverged in numerous nuclear and organelle lineages".]

12 The chemical elements of $s \& p$ types we consider (within this paper) as "intransitive elements", then the elements of $d$ type as "the first transitive elements" and, finaly, the elemets of $f$ type as "the second transitive elements".
} 
elements 2 times and 1 element 4 times; the patterns 9-4-1 and 8-4-2 as unique and very specific mathematical expressions (Eq. 1).

Table 1. Periodic system of chemical elements with 14 lanthanides in 14 groups (Table 4.2 in: Rakočević, 1991, or Table 18 in: Rakočević, 1997b).

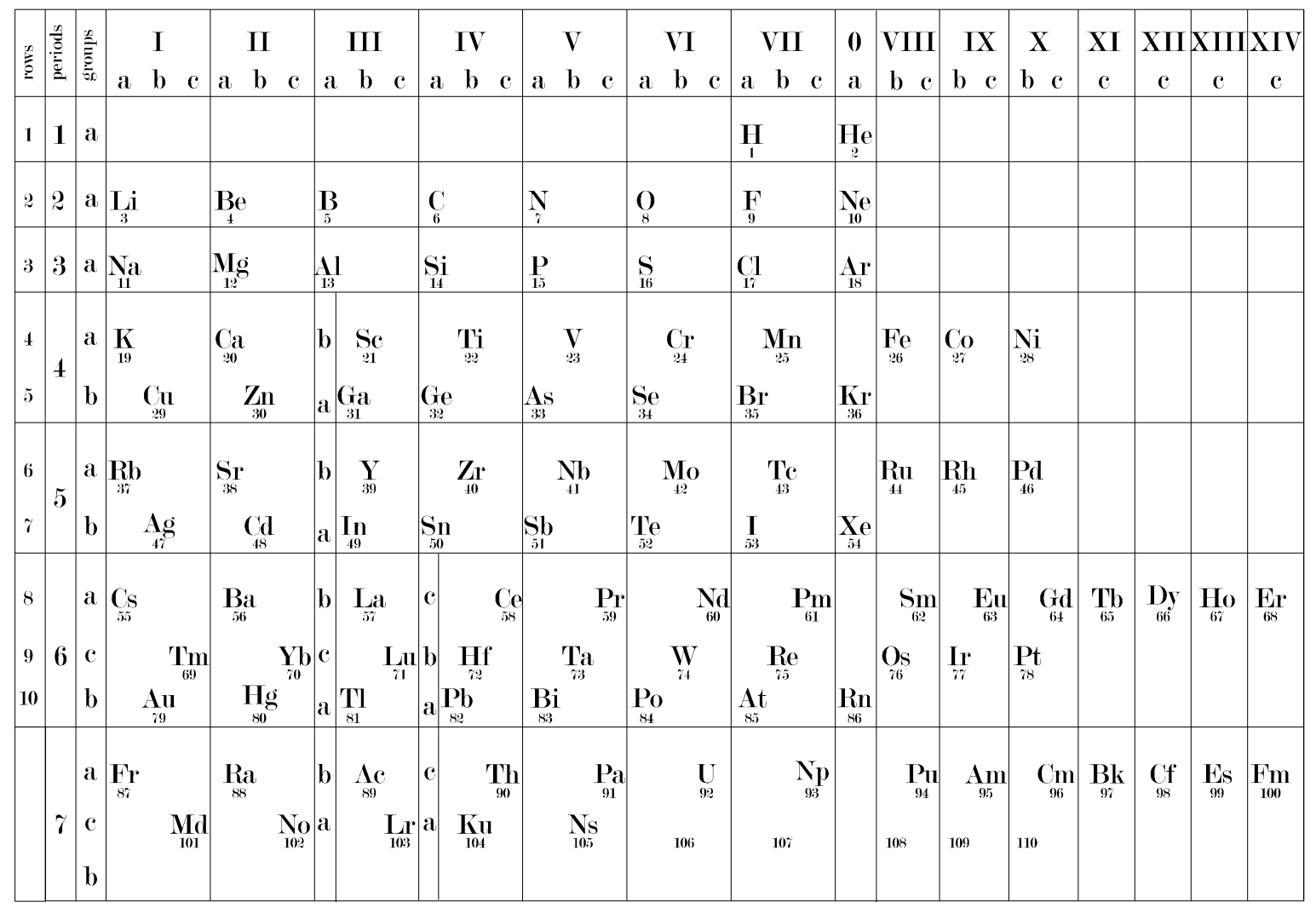

$$
\begin{gathered}
\left(1^{2}+2^{2}+3^{2}=1+4+9\right) /\left(2^{1}+2^{2}+2^{3}=2+4+8\right) \\
\sum_{n=1}^{3} n^{2}=14 \quad \sum_{n=1}^{3} 2^{n}=14 \\
\left(1^{1}+2^{1}+3^{1}=1+2+3\right) /\left(1^{1}+1^{2}+1^{3}=1+1+1\right) \\
\sum_{n=1}^{3} n^{1}=6 \quad \sum_{n=1}^{3} 1^{n}=3
\end{gathered}
$$




\begin{tabular}{|c|c|c|}
\hline $\begin{array}{l}1 . \\
2 .\end{array}$ & $\begin{array}{ll}\left(\mathrm{m}^{1}, \mathrm{~m}^{2}, \mathrm{~m}^{3}\right) ; & (\mathrm{m}=4) \\
\left(\mathrm{n}^{1}, \mathrm{n}^{2}, \mathrm{n}^{3}, \ldots, \mathrm{n}^{6}\right) ; & (\mathrm{n}=2)\end{array}$ & $\begin{array}{l}\text { ["m" as GC alphabet; "n" as binary alphabet }(0, \\
1), \text { valid for the genetic code binary tree. }\end{array}$ \\
\hline 3. & $(\mathrm{~m}=4) ;(\mathrm{n}=2)$ & $\begin{array}{l}{\left[" \mathrm{~m}^{\wedge} \mathrm{n} " \text { as number of doublets in Surv. } 1 ; " \mathrm{n}^{\wedge} \mathrm{m} "\right.} \\
\text { as number of four-codon families (16) in Table } 5 \\
\text { as well as on the genetic code binary tree.] }\end{array}$ \\
\hline
\end{tabular}

Table 2. Periodic system of chemical elements with 6 groups

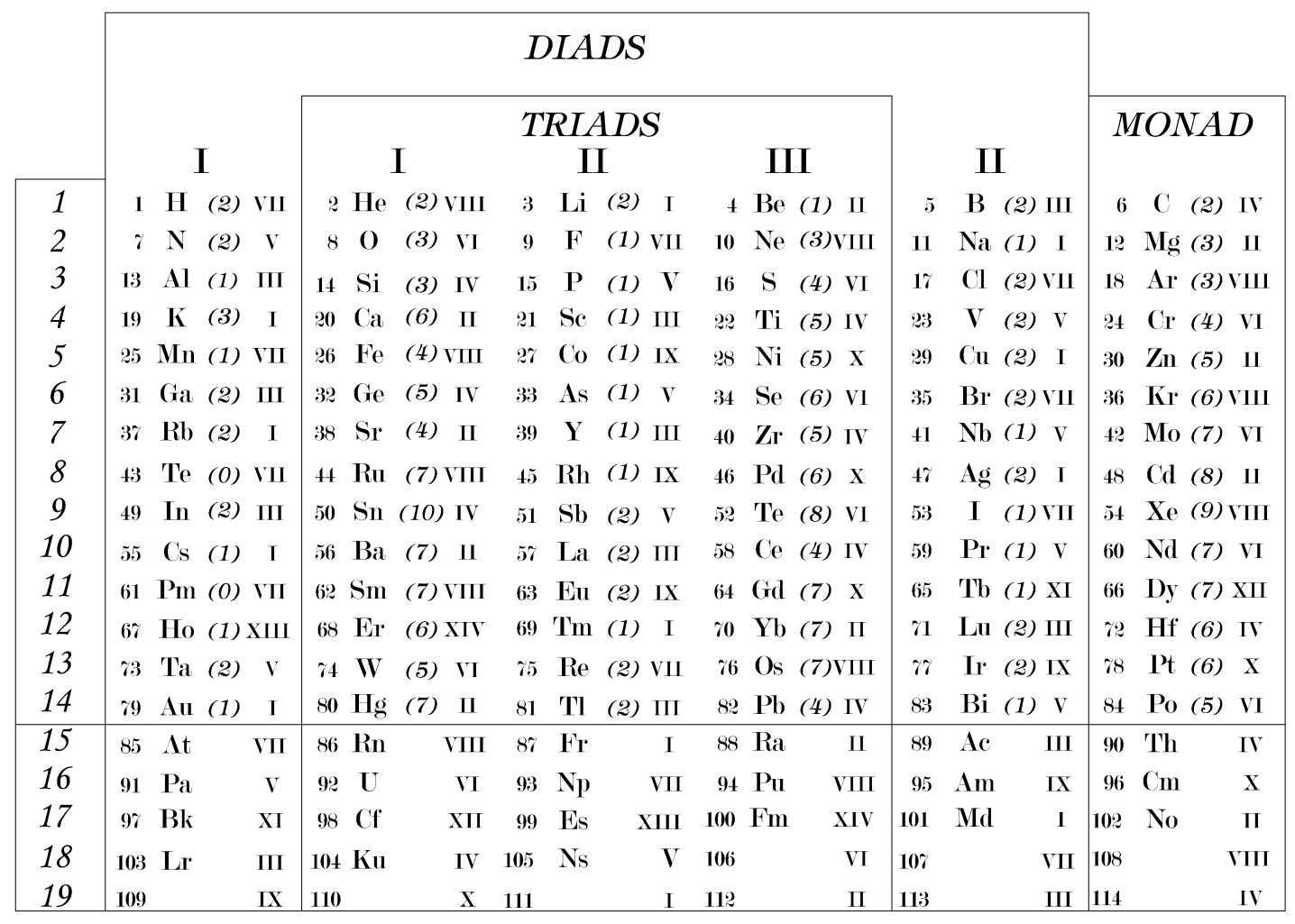

The expression in Eq. (1) ${ }^{14}$ is related to the number of chemical elements in Table 1. On the other hand, the expression (2) is related to triads, diads, monads, respectively, in Table 2 and

\footnotetext{
${ }^{13}$ Through designated exponentiations from "m" follow nucleotide singlets and doublets in Survey 1 , and triplets in Table 5, and from " $n$ " the number of branches on the genetic code binary tree.

${ }^{14}$ From Eq. (1) it follows that the position of the hydrogen in the PSE can only be in the VII group, together with the halogen family, and neither in each other. (Lawrence Sacks, Foundations of Chemistry, 2006, 8, pp. 31-35: “A Coulombic model, in which all compounds of hydrogen are treated as hydrides, places hydrogen exclusively as the first member of the halogen family and forms the basis for reconsideration of fundamental concepts in bonding and structures. The model provides excellent descriptive and predictive ability for structures and reactivities of a wide range of substances.")
} 
Table 3 (in relation to Table 4). In addition one can notice that in reality to the Eq. (1) precedes Eq. (2) as a previous step in exponentiation, valid for the first three natural numbers. The expression in Eq. (3) shows the relationships within the genetic code (cf. Survey 1 and Table 5). As a special case is the set $\left\{\mathrm{m}^{1}, \mathrm{~m}^{2}, \mathrm{~m}^{3}\right\}$ in an indirect correspondence with the set $\left\{2^{1}+2^{2}+2^{3}\right\}$ in Eq. (1) what means the correspondence among genetic code and chemical code. [Eq. (1) corresponds with Table 1; Eq. (2) with Table 2 and Tables 3 \& 4; Eq. (3) with Survey 1 and Table 5.]

Remark 5. Table 1 is essentially a periodic system of short periods. As in the original works of Mendeleev, it can develop into a periodic system of long periods when it per se has a satus of a blockPSE $^{15}$ as a set of adjacent groups: s-block, p-block, d-block and f-block (Rakočević, 1991, Table 4.3 or Rakočević, 1997b, Table 19) (www.rakocevcode.rs). Otherwise, the term "Block Periodic System" as well as "Block Periodic Table" appears to have been first used by Charles Janet, which is understandable (footnote 8), because talking about blocks makes sense only if lanthanides are distributed into 14 groups. Nevertheless, all three of our 1991 Tables where just hypothetical, as it was also the hypothesis that it makes sense to consider PSE as a chemical code. However, now with this new insights into the correspondence with the genetic code, the one confirms the other: without of the PSE Tables of this kind it is not possible to understand the essence of Genetic code, and vice versa - without the insights into the correspondence with the GC we would not have any insight into the existence of a chemical code, which with the genetic code make up a unity, based at the same time on an analogous formalism as well as on a chemical essence.

In Table 2 we actually have the correspondence with the mathematical expression in Eq. (2): 1 set of monads, 1 set of diads, 1 set of triads; at the same time: within the set of monads the isotope number relationships are realized through the singlets of chemical elements; within diads through doublets, and within triads through triplets.

From the $1^{\text {st }}$ to the $8^{\text {th }}$ group, with sub-groups a, b, c, in the PSE (Table 1) are realized the elements correspondingly with the first member of the first mathematical expression in Eq. (1), in the form of $(5+3+1=9)$ elements: 5 intransitive elements $(s$ or $p), 3$ first transitive elements $(d)$ and 1 second transitive element $(f)$. In the final (eighth) case, the zeroth group has the status of $8^{\text {th }}$ group, with 5 intransitive elements. [The group of noble gases has zeroth group status in terms of chemical reactivity, and the status of eighth group in terms of filling the orbitals by electrons.] After the eighth group (with sub-groups b, c) are realized the elements into the ninth and tenth group, corresponding to the second member of the mathematical expression in Eq. (1), in the form of $(3+1)$ : 3 first transitive elements $(d)$ and 1 second transitive element $(f)$. Finally, after tenth group, followed the groups: XI, XII, XIII and XIV, each group with one "second transitive" element $(f)$ correspondent with the third member of the mathematical expression, given in Eq. (1). [Notice that the first three periods are single, each with a single row; fourth and fifth are double, each with two rows and sixth period is threefold - it has three rows.]

Within Table 2 all isotopes (indicated in the brackets) are naturally occurring (stable plus unstable primordial). The exception is Polonium with the number of 'end nuclides', as a result of

\footnotetext{
${ }^{15}$ The block names (s, p, d, f) are derived from the spectroscopic notation for the associated atomic orbitals: sharp,
} principal, diffuse and $\underline{\text { fundamental. }}$ 
radioactive decay of its isotopes existing in the nature in trace amounts, or synthesized: $\mathrm{Pb}-204$, $\mathrm{Pb}-205, \mathrm{~Pb}-206, \mathrm{Bi}-208, \mathrm{Bi}-209$. [This six-groups-PSE one can cf. with the first Mendeleev's Table of PSE, with 6 groups, March 13, 1869, in: Kedrov, 1977, p. 128, photocopy II; also in: Mendelejeff, 1869, reprinted in 1970.]

Let us notice that the arrangement in Table 1 corresponds to the PSE, constituted of 14 groups and 6 periods, and the arrangement in Table 2 corresponds to the PSE constituted of 6 groups and 14 periods. These two PSEs are in correspondance with each other: odd elements are in odd groups, and even element in even groups - in both systems. (Cf. Tables 4.1 \& 4.2 in: Rakočević, 1991, or Tables 18 \& 20 in: Rakočević, 1997b.) (The Reference: Rakočević, 1997b one can see in website: www.rakocevcode.rs)

\subsection{Quantum like relationships within nucleotides arrangements}

The expressions in Eq. (1-3) show the essence of coding / encoding in a natural code; it cannot be reduced only into two alphabets (which are consequences and not the cause). A natural code is such an essence that can be represented by an appropriate mathematical expression, which contains within itself a sequence from the series of natural numbers. From this follows that both, the Genetic code as well as the Periodic system of chemical elements (PSE) are natural codes.

Bearing all this in mind, the expression $\mathrm{N}=\mathrm{n}^{\mathrm{k}}$, valid for the genetic code, is "readable" in the following way: $\mathrm{n}=4 ; \mathrm{k}=3$, where 4 is the number of letters within the GC alphabet, 3 is the number of letters within a word, 2 is the root of the word, and 1 is the number of letters within one letter; altogether, 1, 2, 3, 4, as the sequence of the natural numbers; finely, $\mathrm{N}$ is the number of codons, which encode "letters" from another alphabet, i.e. molecules of AAs. As we see, both Mendeleevian principles are valid, which could not be the case, for example, for $n=5$, when, in the case of validity of Mendeleevian principles, we have uncertainty about the root of the word is it of two or three letters?

The expressions in Eq. (3) show multiple relationships of nucleotide doublets and AAs in Survey 1. But in order to understand these relationships, it is necessary to analyze the relationships between nucleotide doublets arrangement in Survey 1 and arrangement in the original Rumer's Table (Table A. 1); also in order for better understand the sense of our new insight. By comparing two Tables, we find that both in the upper part contain nucleotides with 6 and 4 hydrogen bonds, respectively, that encode AAs, which have 119 atoms in their side chains. In the lower part there are nucleotide doublets with 5 and 5 hydrogen bonds, which encode AAs, which have 120 atoms in their side chains. It can therefore be said that here, in up/down direction, exists a balance with the difference for \pm 1 atom. However, significant differences exist from the aspect of the left/right organization (cf. Survey 1 and Survey A.1).

Remark 6. The number of atoms in the four molecules that build messenger RNA, corresponding to the GCT, is: $\mathrm{U}=12, \mathrm{C}=13, \mathrm{~A}=15, \mathrm{G}=16$; in doublets: $\mathrm{UG}=28 \times 1$ and $\mathrm{CA}=28 \times 1$. When the nucleotide form is taken, then the number of atoms is: $\mathrm{U}=34, \mathrm{C}=35, \mathrm{~A}=37, \mathrm{G}=38$; in doublets: $\mathrm{UG}=$ $36 \times 2$ and CA = 36 × 2. [Cf. (Rakočević, 1997a); (Rakočević, 1997b: Solutions 25-29, pp. 62-63; www.rakocevcode.rs)]. The question arises: is this a curiosity and a coincidence, or is it a certain natural- 
code essence when the sums of the number of atoms per doublets correlate with the square of the first perfect number (6) and the first degree of the second perfect number (28)?

In the Rumer's Table (Table A.1) on the left side are nucleotide doubles that encode fourcodon AAs and on the right side ones that encode non-four-codon AAs. In our Table (Survey 1), however, the nucleotides are mixed; from both types, some of them are on the left and some on the right in original Rumer's Table. Nevertheless, when it looks more carefully, then it can be seen that this is a strictly symmetrical arrangement, a type of mirror symmetry. To more complex "geometric figures" (higher rank!), on the left, correspond less complex "figures" (lower rank!) on the right side: [(UC-CU-GU / CC-GG) // (AU-UA / CA-GA-AG)]; and vice versa: [(UU-AA / $\mathrm{UG}) / /$ (AC / GC-CG)]. But that what is particularly interesting, and in some way very surprising, is the fact that all this "geometry" is accompanied by a "quantum" change for 1 unit in the second position of the decimal number record of the number of atoms: $1 \underline{10} 0$ versus $1 \underline{2} 0$ and 129 versus 119. (For details see Surveys A.1 and A.2).

There is also an additional question - what is the sense of the existence of a link between the left and the right side of the Rumer's Table; the link, expressed through the mirror symmetry and the quantization, presented in Surveys 1 and Surveys A.1, A.2, A.3? The possible respond is: in all here presented arrangements on the scene is a nuance principle in polarity of the amino acid molecules. [For the same reason, some AAs in GCT as well as in the "doublet-triplet" arrangement have been duplicated. $]^{16}$

\begin{tabular}{|c|c|c|c|c|c|c|c|c|c|}
\hline \multicolumn{10}{|c|}{ Survey 1. Generation of 16 nucleotide doublets from four-letter alphabet } \\
\hline \multicolumn{2}{|c|}{$\mathrm{e}=1$} & \multicolumn{6}{|c|}{$\mathrm{e}=2$} & \multicolumn{2}{|c|}{ an } \\
\hline & \multirow[t]{2}{*}{$\mathrm{C}$} & $\mathrm{UU}$ AA $\mathrm{CC}$ & $\mathrm{GG}$ & & $\mathrm{AU}$ & $\mathrm{UA}$ & GC CG & \multirow{3}{*}{60} & \multirow[b]{2}{*}{119} \\
\hline & & $\mathbf{F}, \mathbf{L} \quad \mathbf{N}, \mathbf{K} \quad \mathbf{P}$ & & 59 & $\mathbf{I , M}$ & Y,ct & A & & \\
\hline \multirow[t]{2}{*}{ A } & \multirow[t]{2}{*}{ G } & $\mathrm{UC} \quad \mathrm{CU} \quad \mathrm{GU}$ & UG & & $\mathrm{AC}$ & $\mathrm{CA}$ & GA $\quad \mathrm{AG}$ & & \\
\hline & & $\begin{array}{lll}\mathbf{S} & \mathbf{L} & \mathbf{V} \\
\end{array}$ & $\mathbf{C}, \mathbf{W}$ & 51 & & $\mathbf{H}, \mathbf{Q}$ & $\mathbf{D}, \mathbf{E} \quad \mathbf{S}, \mathbf{R}$ & 69 & 120 \\
\hline \multicolumn{8}{|c|}{110} & \multicolumn{2}{|l|}{129} \\
\hline \multicolumn{4}{|c|}{${ }_{22} \mathbf{U U}_{\mathbf{F L}},{ }_{22} \mathbf{A A}_{\mathbf{N K}},{ }_{33} \mathrm{CC}_{\mathrm{P}},{ }_{33} \mathrm{GG}_{\mathrm{G}}$} & \multicolumn{6}{|c|}{ 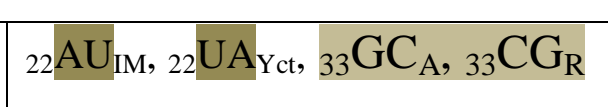 } \\
\hline \multicolumn{4}{|c|}{${ }_{23} \mathrm{UC}_{\mathrm{S}},{ }_{32} \mathrm{CU}_{\mathrm{L}},{ }_{32} \mathrm{GU}_{\mathrm{V}},{ }_{23} \mathrm{UG}_{\mathrm{CW}}$} & \multicolumn{6}{|c|}{${ }_{23} \mathrm{AC}_{\mathrm{T}},{ }_{32} \mathrm{CA}_{\mathrm{HQ}},{ }_{32} \mathrm{GA}_{\mathrm{DE}},{ }_{23} \mathrm{AG}_{\mathrm{SR}}$} \\
\hline
\end{tabular}

\footnotetext{
${ }^{16}$ In the standard GCT the amino acids L, S, R appear twice each; in an extremely nuance reading of the standard GCT the isoleucine appears also twice (Rakočević, 2007b, Table 7; Wohlin, 2015, Table 2); within the "doublettriplet" arrangement of protein AAs where reveals a link between AAs and their biosynthetic precursors, the four AAs from non-alanine stereochemical type (G,P,V,I) appear twice (Survey 1 and Table 1 in Rakočević and Jokić, 1996).
} 
The generation of the 16 nucleotide doublets from four-letter genetic code alphabet, according to the expression $\mathrm{m}^{\wedge} \mathrm{n}(\mathrm{m}=4 ; \mathrm{n}=2$; $\mathrm{e}=$ exponent to alphabet " $\mathrm{m} "$; $\mathrm{an}=$ number of atoms $)$. If $\mathrm{e}=1$, then we have four singlets, the four bases - two pyrimidines (uracil, U; cytosine, C), with one ring in the molecule (chemically simpler), and two purine bases (adenine, A; glutamine, G), with two rings in the molecule (chemically more complex). If $\mathrm{e}=2$, then we have 16 nucleotide doublets. If these doublets are arranged in a chemical hierarchy (first simpler with two hydrogen bonds: UU, AA; then more complex, with three hydrogen bonds: CC, GG; in further steps come their variations, and first row (upper row) is made. These doublets are the same as in upper area of Rumer's Table (Table A.1). Then follows the generation of the lower row of nucleotide doublets, with the idea to present possible mirror symmetry. The result is the discovery of a "hidden" link between two sides of Rumer's Table (Table A.1): of the left side with fourcodon AAs and of the right side with non-four-codon AAs. As it is obvious, the "mirror picture" is expressed through specific "quanta" of the number of atoms in the side chains of AAs as well as through "quanta" of the sets of amino acid molecules (cf. Survey A.1 \& A.2).

Survey 2. Distribution of isotopes within the PSE

\section{(1) Group I:}

$\left\{\left[{ }_{3} \mathrm{Li}(2+0)\right],\left[{ }_{11} \mathrm{Na}(\underline{\mathbf{1}})\right] \&\left[{ }_{19} \mathrm{~K}(2+1)\right],\left[{ }_{37} \mathrm{Rb}(1+1)\right],\left[{ }_{55} \mathrm{Cs}(\underline{\mathbf{1}})\right]\right\}$

$\left\{\left[{ }_{29} \mathrm{Cu}(2+0)\right],\left[{ }_{47} \mathrm{Ag}(2+0)\right],\left[{ }_{79} \mathrm{Au}(\underline{\mathbf{1}})\right]\right\}$

$\left\{\left[{ }_{69} \mathrm{Tm}(\underline{\mathbf{1}})\right]\right\}$

\section{(2) Group II:}

$\left\{\left[{ }_{4} \mathrm{Be}(\underline{\mathbf{1}})\right],\left[{ }_{12} \mathrm{Mg}(3+0)\right] \boldsymbol{\&}\left[{ }_{20} \mathrm{Ca}(5+1)\right],\left[{ }_{38} \mathrm{Sr}(4+0)\right],\left[{ }_{56} \mathrm{Ba}(6+1)\right]\right\}$

$\left\{\left[{ }_{30} \mathrm{Zn}(5+0)\right],\left[{ }_{48} \mathrm{Cd}(6+2)\right],\left[{ }_{80} \mathrm{Hg}(7+0)\right]\right\}$

$\left\{\left[{ }_{70} \mathrm{Yb}(7+0)\right]\right\}$

\section{(3) Group III:}

$\left\{\left[{ }_{5} \mathrm{~B}(2+0)\right],\left[{ }_{13} \mathrm{Al}(\underline{\mathbf{1}})\right] \boldsymbol{\&}\left[{ }_{31} \mathrm{Ga}(2+0)\right],\left[{ }_{49} \mathrm{In}(1+1)\right],\left[{ }_{81} \mathrm{Tl}(2+0)\right]\right\}$

$\left.\left\{\left[{ }_{21} \mathrm{Sc}(\underline{\mathbf{1}})\right],{ }_{39} \mathrm{Y}(\underline{\mathbf{1}})\right],\left[{ }_{57} \mathrm{La}(1+1)\right]\right\}$

$\left\{\left[{ }_{71} \mathrm{Lu}(1+1)\right]\right\}$

\section{(4) Group IV:}

$\left\{\left[{ }_{6} \mathrm{C}(2+0)\right],\left[{ }_{14} \mathrm{Si}(3+0)\right] \&\left[{ }_{32} \mathrm{Ge}(4+1)\right],\left[{ }_{50} \mathrm{Sn}(9+1)\right],\left[{ }_{82} \mathrm{~Pb}(4+0)\right]\right\}$

$\left\{\left[{ }_{22} \mathrm{Ti}(5+0)\right],[40 \mathrm{Zr}(4+1)],\left[{ }_{72} \mathrm{Hf}(5+1)\right]\right\}$

$\left\{\left[{ }_{58} \mathrm{Ce}(4+0)\right]\right\}$

\section{(5) Group V:}

$\left\{\left[{ }_{7} \mathrm{~N}(2+0)\right],\left[{ }_{15} \mathrm{P}(\underline{\mathbf{1}})\right] \boldsymbol{\&}\left[{ }_{33} \mathrm{As}(\underline{\mathbf{1}})\right],\left[{ }_{51} \mathrm{Sb}(2+0)\right],\left[{ }_{83} \mathrm{Bi}(\underline{\mathbf{1}}\right.\right.$ ?) $\left.]\right\}$

$\left\{\left[{ }_{23} \mathrm{Va}(1+1)\right],\left[{ }_{41} \mathrm{Nb}(\underline{\mathbf{1}})\right],\left[{ }_{73} \mathrm{Ta}(2+0)\right]\right\}$

$\left\{\left[{ }_{59} \operatorname{Pr}(\underline{\mathbf{1}})\right]\right\}$

\section{(6) Group VI:}

$\left\{\left[{ }_{8} \mathrm{O}(3+0)\right],\left[{ }_{16} \mathrm{~S}(4+0)\right] \boldsymbol{\&}\left[{ }_{34} \mathrm{Se}(5+1)\right],\left[{ }_{52} \mathrm{Te}(6+2)\right],\left[{ }_{84} \underline{\mathbf{P o}}(5+0)\right]\right\}$

$\left\{\left[{ }_{24} \mathrm{Cr}(4+0)\right],\left[{ }_{42} \mathrm{Mo}(6+1)\right],\left[{ }_{74} \mathrm{~W}(4+1)\right]\right\}$ 
$\left\{\left[{ }_{60} \mathrm{Nd}(5+2)\right]\right\}$

\section{(7) Group VII:}

$\left\{\left[{ }_{1} \mathrm{H}(2+0)\right],\left[{ }_{9} \mathrm{~F}(\underline{\mathbf{1}})\right],\left[{ }_{17} \mathrm{Cl}(2+0)\right] \boldsymbol{\&}\left[{ }_{35} \mathrm{Br}(2+0)\right],\left[{ }_{53} \mathrm{I}(\underline{\mathbf{1}})\right]\right\}$

$\left\{\left[{ }_{25} \mathrm{Mn}(\underline{\mathbf{1}})\right],\left[{ }_{43} \underline{\mathbf{T c}}(0+0)\right],\left[{ }_{75} \operatorname{Re}(1+1)\right]\right\}$

$\left\{\left[{ }_{61} \underline{\mathbf{P m}}(0+0)\right]\right\}$

\section{(8) Groups 0+VIII:}

$\left\{\left[{ }_{2} \mathrm{He}(2+0)\right],\left[{ }_{10} \mathrm{Ne}(3+0)\right],\left[{ }_{18} \mathrm{Ar}(3+0)\right] \boldsymbol{\&}\left[{ }_{36} \mathrm{Kr}(6+0)\right],\left[{ }_{54} \mathrm{Xe}(8+1)\right]\right\}$

$\left\{\left[{ }_{26} \mathrm{Fe}(4+0)\right],\left[{ }_{44} \mathrm{Ru}(7+0)\right],\left[{ }_{76} \mathrm{Os}(6+1)\right]\right\}$

$\left\{\left[{ }_{62} \mathrm{Sm}(5+2)\right]\right\}$

(9) Group IX:

$\{-,-,-,-,-\}$

$\left\{\left[{ }_{27} \mathrm{Co}(\underline{\mathbf{1}})\right],\left[{ }_{45} \mathrm{Rh}(\underline{\mathbf{1}}],\left[{ }_{77} \operatorname{Ir}(2+0)\right]\right\}\right.$

$\left\{\left[{ }_{63} \mathrm{Eu}(1+1)\right]\right\}$

(10) Group X:

$\{-,-,-,-,-\}$

$\left\{[\right.$ [28 $\left.\mathrm{Ni}(5+0)],\left[{ }_{46} \mathrm{Pd}(6+0)\right],\left[{ }_{78} \mathrm{Pt}(5+1)\right]\right\}$

$\left\{\left[{ }_{64} \mathrm{Gd}(6+1)\right]\right\}$

(11) Group XI:

$\{-,-,-,-,-\}$

$\{-,-,-\}$

$\left[{ }_{65} \mathrm{~Tb}(\underline{\mathbf{1}}]\right\}$

(12) Group XII:

$\{-,-,-,-,-\}$

$\{-,-,-\}$

$\left\{\left[{ }_{66} \mathrm{Dy}(7+0)\right]\right\}$

(13) Group XIII:

$\{-,-,-,-,-\}$

$\{-,-,-\}$

$\left\{\left[{ }_{67} \mathrm{Ho}(\underline{\mathbf{1}})\right]\right\}$

(14) Group XIV:

$\{-,-,-,-,-\}$

$\{-,-,-\}$

$\left\{\left[{ }_{68} \operatorname{Er}(6+0)\right]\right\}$ 
To proceed with new insights and analysis some explanations of Survey 2 are necessary as it follows.

I. In front of the sign "+" is the number of stable, while behind the "+" is the number of unstable primordial isotopes.

II. The order within the groups: first come intransitive elements $(s$ or $p),{ }^{17}$ then the first transitive (d), and finally the second transitive element (f). With dark shadow tones are designated the stable elements; a total of 36: 13 odd in the odd groups and 23 even in the even groups (Survey $3 b \& 3 c$ ); with light tones are unstable elements ${ }^{18}$, a total of 25 : the 8 odd in odd groups and the 17 even in even groups (Survey 3b on the left). Unshaded are the 20 stable "monoisotopic" elements (19 odd in odd groups, and 1 even in the even group, $\mathrm{Be}$, in the second one); also unshaded are 3 radioactive elements (Tc, Pm, Po).

III. The presented structure of PSE is characterized by the following regularities:

1. Odd groups contain odd, and even groups contain even elements;

2. Elements of odd groups have 2 or less stable isotopes;

3. Elements of even groups, in short periods have 4 or less, while in long periods have 4 or more stable isotopes;

4. Elements of short periods have only stable isotopes (maximum of $\underline{\mathbf{0}}$ unstable primordial isotopes);

5. Elements of the odd groups of long periods have maximal $\underline{1}$ unstable primordial isotope;

6. Elements of even groups of long periods have maximal $\underline{\mathbf{2}}$ unstable primordial isotopes.

\subsection{Specific algebraic patterns}

Surveys $3 \mathrm{a}, 3 \mathrm{~b}$ and $3 \mathrm{c}$ contain two key results of this research, first on the genetic code, and second one on the chemical code. Survey 3a contains the solutions of the system of two linear algebraic equations (shaded part of Survey), which appear to be in a full accordance with the distribution of codons in the genetic code through coding for 2, 4, 6 and 8 amino acids (Table 5): the 25 codons encode the amino acids of the less complexity (2AAs+4AAs) [(GP)+(ALVI)] which have only carbon and hydrogen (glycine - only hydrogen!) in the side chain; and 36 codons encode the AAs of greater complexity which have, except $\mathrm{C}$ and $\mathrm{H}$, some other elements $(\mathrm{N}, \mathrm{O}$ or $\mathrm{S}$ ). The number of codons for encoding less complex AAs corresponds to the solutions of the first linear equation $\left(\mathrm{x}_{1}=8\right.$ and $\left.\mathrm{y}_{1}=17\right)$ : two nonstandard hydrocarbon AAs (GP) are encoded with 8, and four standard hydrocarbon AAs (ALVI) with 17 codons. On the other hand, the number of codons for encoding more complex AAs corresponds to the solutions of the second linear equation $\left(\mathrm{x}_{2}=10\right.$ and $\left.\mathrm{y}_{2}=26\right)$ : six AAs $(\mathrm{CMFYWH})$ which do not have a mapping of functional groups from the "head" to the "body" (side chain), are encoded with 10, and the eight AAs (STDENQKR), which have a mapping of functional groups from the "head" to the "body" are encoded with 26 codons (Survey 3a).

\footnotetext{
${ }^{17}$ In front of the sign ' $\&$ ' are elements of the short periods, followed by elements of large periods.

${ }^{18}$ Under "unstable elements" we mean those elements, which in addition to the stable, also have unstable primordial isotopes one or more.
} 


\subsection{Key relationhips between GC and PSE}

Now we go to the PSE. The solutions of the system of two linear equations (in the shaded part of Survey 3b) are in an almost wholly accordance with the distribution of chemical elements (in terms of stability/instability and odd/even parity) into periods and groups. From a total of 61 multi-isotope elements, the 25, except stable, possess unstable primordial isotopes (light shaded tones in Survey 2); and 36 multi-isotope elements possess only stable elements (they do not have unstable primordial isotopes) (dark shaded tones in Survey 2).

\begin{tabular}{|c|c|c|c|c|c|}
\hline \multicolumn{2}{|c|}{ Survey 3a } & \multicolumn{4}{|c|}{ Survey 4} \\
\hline $\begin{array}{l}y_{1}-x_{1}=3^{2} \\
y_{1}+x_{1}=5^{2}\end{array}$ & $\begin{array}{l}y_{2}-x_{2}=4^{2} \\
y_{2}+x_{2}=6^{2}\end{array}$ & \multicolumn{4}{|c|}{$\begin{array}{ll}26=X=\mathbf{2 6} & 16+17+18=Z \\
26+16=\mathbf{4 2} & Z=51 \\
42+17=\mathbf{5 9} & Z=Y / 4 \\
59+18=77 & \end{array}$} \\
\hline$x_{1}=8$ & $\begin{array}{l}x_{2}=10 \\
y_{2}=26\end{array}$ & \multicolumn{4}{|c|}{$\begin{array}{l}26+42+59+77=\mathrm{Y} \\
\mathrm{Y}=204 ; \mathrm{Y} / 4=51\end{array}$} \\
\hline$y_{1}=17$ & & \multicolumn{4}{|c|}{ In any other case $\mathrm{Y} / 4 \neq \mathrm{Z}$} \\
\hline \multicolumn{2}{|c|}{ Survey 3b } & \multicolumn{4}{|c|}{ Survey 3c } \\
\hline $\begin{array}{c}y_{1}-x_{1}=9^{1} \\
y_{1}+x_{1}=25^{1}\end{array}$ & $\begin{array}{l}y_{2}-x_{2}=\mathbf{1 0}^{1} \\
y_{2}+x_{2}=6^{1}\end{array}$ & $\begin{array}{l}\mathrm{a} \\
00 \\
01 \\
02 \\
03\end{array}$ & $\begin{array}{l}\mathrm{b} \\
\mathbf{1 0} \\
11 \\
12 \\
13\end{array}$ & $\begin{array}{c}c \\
26 \\
25 \\
24 \\
23\end{array}$ & $\begin{array}{l}d \\
(16) \\
(14) \\
(12) \\
(10)\end{array}$ \\
\hline $\begin{array}{l}x_{1}=8 \\
y_{1}=17\end{array}$ & $\begin{array}{l}x_{2}=13 \\
y_{2}=23\end{array}$ & $\begin{array}{l}03 \\
04 \\
05 \\
06\end{array}$ & $\begin{array}{l}13 \\
14 \\
15 \\
16\end{array}$ & $\begin{array}{l}23 \\
22 \\
21 \\
20\end{array}$ & $\begin{array}{l}(\underline{(0)}) \\
(08) \\
(06) \\
(04)\end{array}$ \\
\hline
\end{tabular}

The systems of linear equations as the scenarios for generating GC and PSE are given in Survey 3a and $3 \mathrm{~b}$, respectively; in Survey $3 \mathrm{c}$ there is an additional scenario for PSE with the showing why $\mathrm{x}_{2}=13$ and $\mathrm{y}_{2}$ $=23$; that comes from the fact that only in the fourth row of Surveys $3 \mathrm{c}$ we have $(03,13,23)$, a determination with the principles of similarity and self-similarity; columns: $a=b-10$ and $d=c-b$; columns "b" and "c" correspond to $\mathrm{x}_{2}$ and $\mathrm{y}_{2}$, respectively. [Survey 4 comes from Fig. 3 and Table 4 in Rakočević, 2011b. (Other details in the text).]

Further distributions are carried out through distinctions into odd and even elements - the odd elements within the odd groups and the even elements within the even groups, in both cases are in accordance with the model (the shaded part in Survey $3 \mathrm{~b}$ ). In accordance with the solutions of the first linear equations of $\left(\mathrm{x}_{1}=8\right.$ and $\left.\mathrm{y}_{1}=17\right)$, the 8 unstable and odd elements are within the odd, and 17 unstable and even elements within the even groups. On the other hand, 
according to the solutions of the second linear equation $\left(x_{2}=13\right.$ and $\left.y_{2}=23\right)$, the 13 stable and odd elements are in odd and 23 stable and even elements in even groups. [Under the notion "unstable" we mean the chemical element, which in addition to stable isotopes, have at least one unstable primordial isotope.]

\subsection{Some additional observations on Genetic code}

At the end of the presentation of these research-insights-results, we once again return to the genetic code. Let us look at Table 3 and Table 4, in relation to Eq. (4). In Table 3 we have: monads - the numbers that appear at once; diads - twice, and triads - three times. At dark tones are the amino acids handled by enzymes of class I aminoacyl-tRNA synthetases and at light tones the amino acids handled by enzymes of class II aminoacyl-tRNA synthetases. [One can notice here a correspondence with PSE in Table 2 and with the mathematical expression in Eq. (2).]

The algorithm for "selection" of numbers in Tab. 4, going from bottom is as follows: in the first step were selected 2 numbers going up, and $\underline{\mathbf{0}}$ numbers going sideways, that is 2 vs $\underline{\mathbf{0}}$ (17 \& 18 versus nothing \& nothing); in the second step: 2 vs $\underline{\mathbf{2}}$ (7\& 14 versus $13 \& 15)$; third step: 2 vs $\underline{4}(1 \& 4$ versus $5 \& 10$ and $8 \& 11$ ); in the fourth step would be 2 vs $\underline{\mathbf{6}}$, etc. (Eq.4).

In Table 4 we actually have the relationships within the atom number arrangement in Table 3, which arrangement corresponds with the mathematical expression in Eq. (2) in the next sense: 1 set of monads, 1 set of diads, 1 set of triads; at the same time: within the set of monads each number appears just once, within the set of diads - twice, and within the set of triads - three times. On the other hand, the "choices" presented in Eq. (4) are agreed with a specific algorithm which corresponds with Generalized Golden Mean (GGM), through "metallic means family", for $q=2,6,12,20, \ldots$ (Rakočević, 2004b, p. 97).

With first choice there are $\underline{\mathbf{2}}$ number-patterns: $18 \& 17$; with the second one there are $\underline{\mathbf{6}}$ number-patterns: $(18 \& 17)+[(14 \& 07)+(13 \& 15)]$; with the third choice there are $\underline{\mathbf{1 2}}$ number-patterns: $(18 \& 17)+[(14 \& 07)+(13 \& 15)]+[(04 \& 01)+(05 \& 10)+(08 \& 11)]$. With this third choice there are all number-patterns for the number of atoms in amino acid molecules, within their side chains (12 patterns in Table 4 follow from 20 numbers, presented in Table 3). By all this, we can notice a regularity, valid for the GGM series, expressed in Eq. (4) and related to Eq. (5).

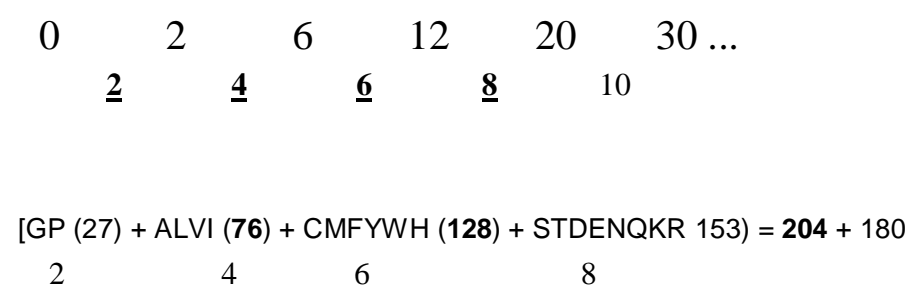

Remark 7. Within four diversity types of 2-4-6-8 AAs there is the number of atoms as follows. Within the "heads" 180 atoms: I.GP 18; II.ALVI 36; III.CMFYWH 54; IV.STDENQKR 72); within the 
side chains 204 atoms: I.GP 9; II.ALVI 40; III.CMFYWH 74; IV.STDENQKR 81; in total, as in Eq. (5) where we have an example of self-similarity: in two inner groups, with 10 AAs, there are 204 atoms as in 20 side chains. On the other hand, in two outer groups, also with 10 amino acids, there are 180 atoms as in 20 amino acid heads. There is also a significant relation to the system, presented in Survey 4: [I.GACNP (26); II.SDTQH (42); III.YMEVL (59); IV.WRFIK (77)], which system follows from a unique arithmetical arrangement of natural numbers from 01 to 99 (Table 4 in relation to Figure 3 in Rakočević, 2011b).

Remark 8. If the Multiplication table in the decimal numbering system is written in an adequate way (Table 1.1 in: Rakočević, 2011b, p. 822) then the numbers per diagonal: $0,2,6,12,20,30$, ..., are also recognized as values for $q$ in the equation of the Generalized golden mean $\mathrm{x}^{2}+p \mathrm{x}=q(q=0,2,6,12,20$, $30, \ldots)$ in the set of the family of "metallic means" (Spinadel, 1998, 1999; Rakočević, 2004b). The solutions of equations in such cases are generated from a set of natural numbers: $(0,1),(1,2),(2,3),(3$, 4), $(4,5), \ldots$; [First family of "metallic means": "golden mean" ( $\mathrm{p}=1 ; \mathrm{q}=1)$; "silver mean" ( $\mathrm{p}=2 ; \mathrm{q}=1)$; "bronze mean" ( $\mathrm{p}=3 ; \mathrm{q}=1), \ldots$; the second family of "metallic means": "golden mean" $(\mathrm{p}=1 ; \mathrm{q}=1)$; "copper mean" ( $\mathrm{p}=1 ; \mathrm{q}=2)$, "nickel mean" $(\mathrm{p}=1 ; \mathrm{q}=3, \ldots)$.]

Remark 9. Double values of the numbers found on the diagonal of Multiplication table: $0,4,12,24,40$, $60, \ldots$, are also recognized as values of one of the cathetus of Diophantus' triangles: $0 .(1, \underline{0}, 1), 1 .(5, \underline{4}, 3), 2$. $(13, \underline{12}, 5), 3$. $(25, \underline{24}, 7), 4$. $(41, \underline{40}, 9), 5 .(61, \underline{60}, 11), \ldots$, etc. Knowing above presented connections with the generalized golden mean, and that the genetic code is also determined by the golden mean (Rakočević, 1998b), we understand the finding of V. Shcherbak, according to which the coding of four-codon amino acids "goes" over the Pythagorian triplet (5, 4, 3) (Shcherbak, 1994).

Remark 10. From the fact that the logic of the choices of the quantities of the number of atoms corresponds with the number sequence in Eq. (4), and this sequence $(0,2,6,12,20 \ldots)$ corresponds to the generalized golden mean and Diophantus' triangles at the same time, it follows that the choice of the quantitees of the number of atoms is also determined by both - by the generalized golden mean and by the set of Diophantus' triangles. [Quantities of the number of atoms: all different in monads; more quantities are the same in diads, as well as in triads, in Table 3.]

Remark 11. From the fact that Eq. (4) corresponds with Eq. (5) through the sequence of the first even numbers $(2,4,6,8)$, from the series of natural numbers, it follows that classification of amino acids into presented four classes is also determined by both - by the generalized golden mean and by the set of Diophantus' triangles.

Table 3. The number of atoms in the protein AAs (in their side chains)

\begin{tabular}{|c|c|c|c|c|c|}
\hline \multirow{2}{*}{\begin{tabular}{|l} 
Monads \\
G 01
\end{tabular}} & \multicolumn{2}{|c|}{ Diads } & \multicolumn{3}{|c|}{ Triads } \\
\hline & C 05 & S 05 & T 08 & P 08 & N 08 \\
\hline A 04 & V 10 & E 10 & Q 11 & M 11 & $\mathrm{H} 11$ \\
\hline D $\quad 07$ & L 13 & I 13 & & & \\
\hline $\mathrm{F} \quad 14$ & Y 15 & K 15 & & & \\
\hline R 17 & & & & & \\
\hline W 18 & & & & & \\
\hline
\end{tabular}

(Explanation in the text) 
Table 4. The relationships to the atom number arrangement in Table 3

\begin{tabular}{|ccc|cc|}
\hline $\mathbf{1}$ & $\mathbf{2}$ & $\mathbf{3}$ & & \\
\hline 01 & 05 & 08 & $\rightarrow$ & $\mathbf{3}$ \\
04 & 10 & 11 & $\rightarrow$ & $\mathbf{3}$ \\
07 & 13 & - & $\rightarrow$ & $\mathbf{2}$ \\
14 & 15 & - & $\rightarrow$ & $\mathbf{2}$ \\
17 & - & - & $\rightarrow$ & $\mathbf{1}$ \\
18 & - & - & $\rightarrow$ & $\mathbf{1}$ \\
\hline $\mathbf{6}$ & $\mathbf{4}$ & $\mathbf{2}$ & $(12)$ \\
\hline $\mathbf{6}$ & $\mathbf{8}$ & $\mathbf{6}$ & $(20)$ \\
\hline
\end{tabular}

Table 5. The standard genetic code with new design

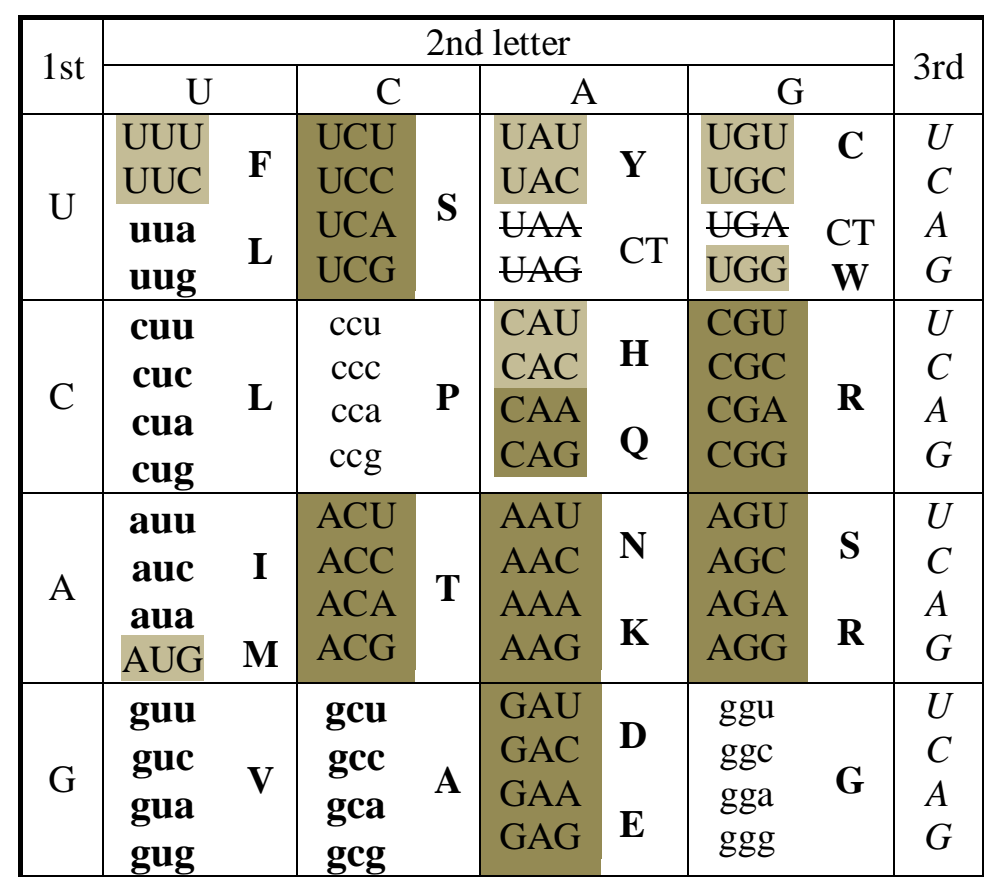

The design responds to the classification of protein AAs into four classes, correspondently with four diversity types. The first diversity type (GP): the 8 codons in small non-bolding letters; second type (ALVI), the 17 codons in small bolding letters; third type (CMFYWH), the 10 codons in large letters and light shadow tones; fourth type (STDENQKR): the 26 codons in large letters and dark shadow tones. The three codons which are cross out, are the "stop" codons (cf. Survey 3a and Survey 4 in this paper with illustrations in Rakočević, 2011b: Tab. 4, Eq. 3 and Eq. 4 on pp. 826-827; Table. 6 on p. 829). 


\title{
3. Discussion
}

For discussion of the obtained results, it is worth mentioning the current understanding of isotopy science, and hence we quote the comment of IUPAC, supplied with the official "Periodic Table of Isotopes":

\begin{abstract}
"Standard atomic weights are the best estimates by IUPAC of atomic weights that are found in normal materials, which are terrestrial materials that are reasonably possible sources for elements and their compounds in commerce, industry, or science. They are determined using all stable isotopes and selected radioactive isotopes (having relatively long half-lives and characteristic terrestrial isotopic compositions). Isotopes are considered stable (non-radioactive) if evidence for radioactive decay has not been detected experimentally" (IUPAC Project 2007-038-3-200, "Development of an isotopic periodic table for the educational community", October 1, 2013, www.ciaaw.org). ${ }^{19}$
\end{abstract}

It is understood that the discussion of isotopes of chemical elements within the scientific community is strictly limited to planet Earth, because that is what we do know from the experiments. However, if it is possible to find a theoretical model, an arithmetic-algebraic scenario, that is, at a given moment of evolution of scientific knowledge, agreed with the distribution of chemical elements in the PSE, in terms odd-even parity of elements and their isotopes stability (moreover, agreed with corresponding distributions in the GC), then it should be that this model is valid for the whole universe, due to the fact that the content of the universe consists of one and the same type of chemical elements and their isotopes.

However, having in mind the fact that the stability/instability of isotopes is experimentally determined (certainly by the best and most reliable laboratories, in terrestrial conditions), it must be said that the presented accordance with the models in Surveys 3a, $3 \mathrm{~b}$ and $3 \mathrm{c}$, although one hundred percent, or near to be one hundred percent, it is only one very distinctive trend, which should serve for further researches in both directions: for checking experimental results, and parallel with this, for checking the accordance with the models presented in Surveys 3a, 3b and $3 c$.

In the current state of affairs, what may possibly spoil a 100-percent accordance, it might be in the next. In relevant literature one can see that potentially three of ten isotopes of tin are radioactive, but have not been observed to decay. One of these three actually has been identified as Sn-124, and in IUPAC sources is indicated its half-life decay larger than $1 \times 10^{17}$ years. In addition, one of the seven (stable) isotopes of Gadolinium (Gd-152) we take to be unstable with a half-life decay of $1.08 \times 10^{14}$ years, while the isotope (Gd-160) with a half-life decay larger than $1.3 \times 10^{21}$ remained in a stable status. Overall, we marked the isotopic state of Gadolinium

\footnotetext{
19 "Dec 2016 - Project update published in Chem. Int. Nov 2016, p. 25; https://doi.org/10.1515/ci-2016-0619 (Page
} last updated 11 Jan 2017)" 
$(6+1)$. These are only two our interventions in relation to the IUPAC document which are, as follows from the above, also consistent with the understanding of isotopy in current science. ${ }^{20}$

\subsection{Another understanding of the periodic system}

For an additional understanding of the PSE shown in Table 1 in the form of 6 periods and 14 groups, it is necessary to include a specific variant of the PSE which is inversely related in the sense that it consists of 14 periods and the 6 groups (Table 2).

The arrangement of elements in the PSE in Table 2 is such that the odd elements are in odd and even elements in even groups, in the same way as in the PSE presented in Table 1. From this it follows that the same regularities, indicated in the explanations in Survey 2 (Section III), are valid for both, PSE in Table 1 as well as in Table 2. In addition, here we also find additional regularities. For them it is necessary to note that the arrangement of the PSE in Table 2 can be read in two ways: first, as indicated monads, diads and triads of columns, i.e. groups; and second, as odd and even groups (I, II, III, IV, V, VI). Correspondent comparisons (the left column of diads vs right column; left column of triads vs right; then, the first column vs third, the second vs fourth, etc.) show that the trend in differences, in the number of naturaly occurring isotopes, is such, that these differences, in 10 or more cases (from total 14), is less than 2. A maximum of two times, there are differences of 2 or 3 isotopes; altogether, all differences are within the frame of the set $\{0,1,2,3\}$, that is of a "logical square".

\subsection{Another similarities}

From Table 4 it is self-evident that here we have two solutions: the number of moleculespatterns in 20 canonical AAs is 6-4-2 as the sum of number-patterns in monads-diads-triads; on the other hand real sum of amino acid molecules is $6+8+6=20$. The pattern ,686" corresponds to the number 686 in decimal numbering system, which is the number of protons in 20 amino acids (within their side chains), what means a similarity and self-similarity. If we exclude the number of hydrogen atoms, that is hydrogen protons $(58+59=117)$ of all 20 side chains, then remain 569 protons +569 neutrons. At the same time, from Sukhodolets' system (Table C.2 in this paper in relation to Table 7, p. 830 in Rakočević, 2011b) follows a symmetrical division of AAs into two sub-classes with 58 and 59 hydrogen atoms, respectively.

\subsection{Some additional observations}

The simplest chemical elements as nonmetal atoms, in the PSE, are:

$$
\mathrm{H}+\mathrm{C}+\mathrm{N}+\mathrm{O} \Rightarrow 4 \text { simplest nonmetal atoms }
$$

Their simplest compounds are methane, ammonia, water and carbon monoxide:

\footnotetext{
${ }^{20}$ In IUPAC document - IUPAC Project 2007-038-3-200, "Development of an isotopic periodic table for the educational community" (October 1, 2013 www.ciaaw.org), for Europium was indicated the state $(2+0)$, what means that both its isotopes are stable. However, in "New interactive, electronic version of the IUPAC Periodic Table of the Elements and Isotopes" it is modified so that now it is EU $(1+1)$ because EU-151 is unstable and EU153 the stable isotope, as it is in our system in Survey 2 [Eu-63 (1+1)].
} 


$$
\mathrm{CH}_{4}+\mathrm{NH}_{3},+\mathrm{H}_{2} \mathrm{O}+\mathrm{CO} \Rightarrow 4 \text { related simplest molecules }
$$

Their functional groups are:

$$
\mathrm{CH}+\mathrm{NH}_{2},+\mathrm{OH}+\mathrm{CO} \Rightarrow 9 \text { atoms }
$$

The first possible organic molecule which takes into account all these groups is the simplest possible amino acid - glycine.

Then, we ask the Shcherbak's "mathematical trace" for nucleon number in 9 atoms:

$$
\mathrm{CH} 13+\mathrm{NH}_{2} 16+\mathrm{OH} 17+\mathrm{CO} 28=74(2 \times 37)
$$

So much, in fact, there are nucleons in the "head" of amino acid (35 protons and 39 neutrons).

To better understand the above presented facts and analogies, in a future research it would be also the worth to take into account that both, the PSE and the GC, satisfy the golden mean property. ${ }^{21}$ [The GC satisfies the golden mean, over the set of all atoms (Rakočević, 1998b, 2011b) as well as through the number of carbon atoms in 20 amino acids (Yang, 2000).] This should in particular be kept in mind for further testing "a new standpoint for addressing questions of selection vs random drift in the evolution of the code" (Swanson, 1984).

\section{Conclusion}

Considering the periodic system of chemical elements as a chemical code, then one can observe certain analogies between this code and the genetic code. This paper is just devoted to analogies between these two codes. The presented research-insights-results show that the key to these analogies are specific relationships. Namely, within the genetic code there are exactly 61 codons wich encoding amino acids, plus 3 stop codons, plus 20 protein amino acids. On the other hand, within the chemical code there are exactly 61 entities in the form of stable isotopes, plus 3 unstable isotopes (Technetium, Promethium and Polonium), plus 20 ,non-isotope“ entities (20 "monoisotope" elements). Thus, altogether there are 84 entities within the genetic as well as 84 entities within the chemical code.

Moreover, the obtained analogies contain also a deeper connection between the genetic code and the periodic system of chemical elements. For example, inside 61 codons 25 of them encode the amino acids of less complexity (GP+ALVI), and 36 codons encode the AAs of greater complexity (CMFYWH+STDENQKR). Analogies can be further expanded by splitting less complex and more complex sets of AAs into subsets (indicated in parentheses). The distribution of codons is related to solutions of the system of two linear equations presented in the upper part of Survey 3a (8 codons for GP and 17 codons for ALVI equals 25 codons; 10 codons for CMFYWH and 26 codons for STDENQKR equals 36 codons) (Table 5).

Analogies with chemical code are as follows. From total 61 multi-isotope elements, 25 of them, except stable, possess unstable primordial isotopes; in accordance with the solutions of the first linear equation $\left(\mathrm{x}_{1}=8\right.$ and $\left.\mathrm{y}_{1}=17\right)$, in Survey $3 \mathrm{a}$, where the 8 unstable and odd elements are within the odd, and 17 unstable and even elements within the even groups. On the other hand,

\footnotetext{
${ }^{21}$ About Golden mean in periodic system, see in: Luchinskiy \& Trifonov, 1981; Rakočević, 1998a. About Golden mean in GC see in: Rakočević, 1998b, 2014.
} 
according to the solutions of the second linear equation $\left(x_{2}=13\right.$ and $\left.y_{2}=23\right)$, in Survey $3 b$, where the 13 stable and odd elements are in odd and 23 stable and even elements in even groups.

In addition to the analogies of the number of isotopes, and the number of codons, there is an analogy more through the number of the chemical elements (in the arrangement of PSE with 6 groups - Table 2) and the number of atoms in the 20 protein AAs (Table 4): in both cases the order is made through the monads, diads and triads - with the singlets, doublets and triplets, respectively.

At the end, there is a sense to assume that regularities in the genetic code are caused, at least partially, by regularities in the periodic system of chemical elements.

\section{Acknowledgement}

I am grateful to Branko Dragovich, Vladimir Ajdačić and Nataša Mišić for support and helpful, stimulating discussion. 


\section{Appendix A. Two amino acid classes: four-codon and non-four-codon AAs}

Table A.1. The Rumer's classification of amino acids into two classes

\begin{tabular}{|c|c|c|c|c|}
\hline 114 & $\begin{array}{c}30 \\
116\end{array}$ & (119) & $\begin{array}{c}89 \\
108\end{array}$ & 125 \\
\hline $\begin{array}{l}\text { Gly } \\
\text { Pro } \\
\text { Arg } \\
\text { Ala }\end{array}$ & $\begin{array}{l}\text { GG (6) } \\
\text { CC (6) } \\
\text { CG (6) } \\
\text { GC (6) }\end{array}$ & $\begin{array}{l}\text { Phe } \\
\text { Asn } \\
\text { lle } \\
\text { Tyr }\end{array}$ & $\begin{array}{l}\text { UU (4) } \\
\text { AA (4) } \\
\text { AU (4) } \\
\text { UA (4) }\end{array}$ & $\begin{array}{l}\text { Leu } \\
\text { Lys } \\
\text { Met } \\
\text { ct }\end{array}$ \\
\hline $\begin{array}{l}\text { Thr } \\
\text { Val } \\
\text { Ser } \\
\text { Leu }\end{array}$ & $\begin{array}{l}\text { AC (5) } \\
\text { GU (5) } \\
\text { UC (5) } \\
\text { CU (5) }\end{array}$ & $\begin{array}{l}\text { His } \\
\text { Cys } \\
\text { Asp } \\
\text { Ser }\end{array}$ & $\begin{array}{l}\text { CA (5) } \\
\text { UG (5) } \\
\text { GA (5) } \\
\text { AG (5) }\end{array}$ & $\begin{array}{l}\text { Gln } \\
\text { Trp } \\
\text { Glu } \\
\text { Arg }\end{array}$ \\
\hline 125 & $\begin{array}{c}36 \\
106\end{array}$ & (120) & $\begin{array}{c}84 \\
118 \\
\end{array}$ & 114 \\
\hline & $0-66$ & & $330 \pm 00$ & \\
\hline \multicolumn{5}{|c|}{$\begin{array}{c}125+114=239 \\
125-114=\mathbf{1 1}\end{array}$} \\
\hline
\end{tabular}

The four-codon amino acids are on the left and the non-four-codon amino acids on the right. Each of two classes is classified into two subclasses, corresponding to the number of hydrogen bonds in the nucleotide doublets. At the bottom (shaded) - the number of atoms in the amino acid molecules (side chains): within 32 amino acid molecules on the left and 29 on the right, within the set of "61" amino acid molecules, each molecule encoded by one codon. [There are the same results, 330-66 (as $8 \times 33$ ) and 330 (as $10 \times 33$ ) in standard GCT, both in relation with the result $(9 \times 33) \pm 1$ as we have shown in a previous work (Rakočević, 2004a, Table 3a on p. 224).] Up/down: 119/120 atoms in 11/12 amino acids (side chains), respectively, within the set of "23" amino acids. [Calculations: $(30+89=119) ;(36+84=120) ;(30+84=$ 114); $(36+89=125)$.] Up/down: $(116 / 1 \underline{08} / / 106 / 1 \underline{18})$ as the number of atoms within nucleotide doublets. (Note: In originsl Rumer's Table only the number of hidrogen bonds is calculated; all other calculations are ours.) 
Table A.2. The Shcherbak's Table of multiples of "Prime quantum 037" (Table 1 in: Shcherbak, 1994).

\begin{tabular}{|ccccccccc|}
\hline 1 & 2 & 3 & 4 & 5 & 6 & 7 & 8 & 9 \\
037 & 074 & 111 & 148 & 185 & 222 & 259 & 296 & 333 \\
10 & 11 & 12 & 13 & 14 & 15 & 16 & 17 & 18 \\
370 & 407 & 444 & 481 & 518 & 555 & 592 & 629 & 666 \\
19 & 20 & 21 & 22 & 23 & 24 & 25 & 26 & 27 \\
703 & 740 & 777 & 814 & 851 & 888 & 925 & 962 & 999 \\
\hline
\end{tabular}

The Shcherbak's basic Table indicates that the determination of the number of nucleons within amino acid molecules occurs by the multiples of number 037 (as a "Prime Quantum"), by the numbers with the same digits $(111,222,333$ etc.) or by the permutations of the obtain different cipher multiples.

Survey A.1. Relationships between AAs in Rumer's Table in accordance with the quantumblock "Aufbau" principle

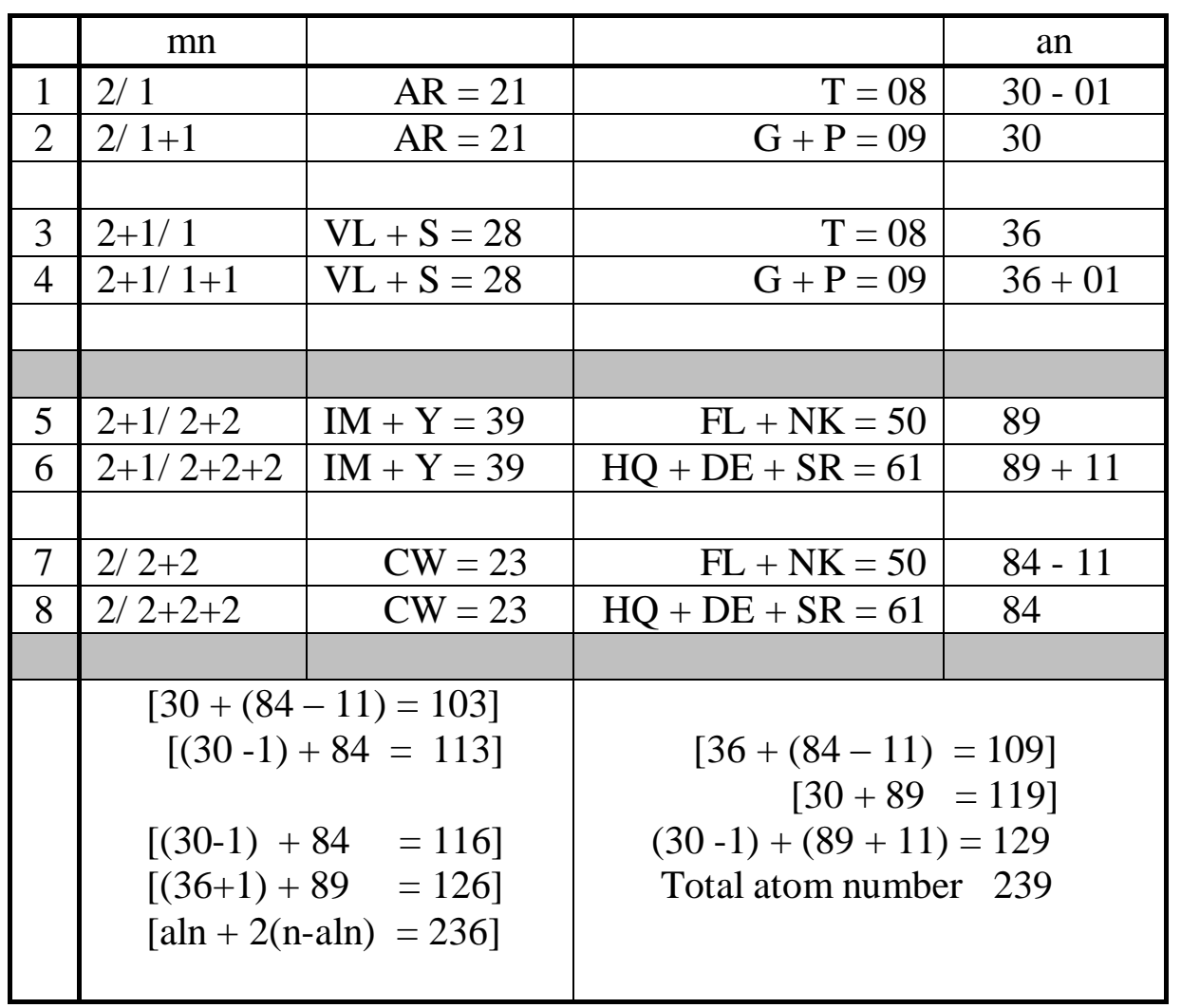

In the first four rows there are four-codon AAs (from the left side in Table A.1) and in the second four rows non-four-codon AAs (from the right side in Table A.1). In the first case, the "blocks" of AAs differ by 01 atom, and in the second case by 11 . The designations: $\mathrm{mn}$ - number of molecules; an - number of atoms; at the bottom: "aln" for AAs of alanine stereochemical type; "n-aln" for 
AAs of non-alanine stereochemical types (Rakočević \& Jokić, 1996); "Total atom number" - the number of atoms within 23 AAs (side chains) in standard GCT.

Survey A.2. Relationships between Rumer's Table in Table A-1 and our Table in Survey 1

\begin{tabular}{|c|c|c|c|c|}
\hline 1 & $\begin{array}{l}\text { Quantum "30 - 01" } \\
\text { (ac) T (dn, right) } \\
\text { (gc, cg) A, R (up, right) }\end{array}$ & 6 & $\begin{array}{l}\text { Quantum "89+11" } \\
\text { (ca, ga, ag) HQ, DE, SR (dn, right) } \\
\text { (au, ua) IM, Y (up, right) }\end{array}$ & 129 \\
\hline 2 & $\begin{array}{l}\text { Quantum "30" } \\
\text { (gg, cc) G, P (up, left) } \\
\text { (gc, cg) A, R (up, right) }\end{array}$ & 5 & $\begin{array}{l}\text { Quantum "89" } \\
\text { (uu, aa) FL, NK (up, left) } \\
\text { (au, ua) IM, Y (up, right) }\end{array}$ & 119 \\
\hline 3 & $\begin{array}{l}\text { Quantum "36" } \\
\underline{(\mathrm{ac}) \mathrm{T}(\mathrm{dn}, \text { right })} \\
(g u, c u, u c) V, L, S(d n, l e f t)\end{array}$ & 8 & $\begin{array}{l}\text { Quantum "84" } \\
(\mathrm{ca}, \mathrm{ga}, \mathrm{ag}) \mathrm{HQ}, \mathrm{DE}, \mathrm{SR}(\mathrm{dn}, \text { right }) \\
(\text { ug) } C W(d n, \text { left })\end{array}$ & 120 \\
\hline 4 & $\begin{array}{l}\text { Quantum "36+ 01" } \\
(\mathrm{gg}, \mathrm{cc}) \mathrm{G}, \mathrm{P}(\mathrm{up}, \mathrm{left}) \\
(g u, c u, u c) V, L, S(d n, \text { left })\end{array}$ & 7 & $\begin{array}{l}\text { Quantum "84 -11" } \\
(\text { uu, aa) FL, NK (up, left) } \\
(u g) C W(d n, \text { left) }\end{array}$ & 110 \\
\hline
\end{tabular}

The whole arrangement is in relation to the arrangement of AAs in Survey A.1. On the left are four-codon AAs and on the right non-four-codon AAs (as in Table A.1). The designations "up", "dn" (down), "left" and "right" refer to the positions in Survey 1. Amino acids at the positions indicated by the numbers 1-8 correspond to the amino acids at the same positions in Survey A-1. 
Survey A.3. Relationships between the rows in Survey A.1

\begin{tabular}{|l|lll|l|l|}
\hline$(1-6)$ & $(30-1)+(89+11)$ & $=$ & 129 & & \\
$(1-5)$ & $(30-1)+(89+00)$ & $=$ & $\underline{\mathbf{1 1 8}}$ & & \\
$(2-6)$ & $(30-0)+(89+11)$ & $=$ & 130 & & \\
$(2-5)$ & $(30-0)+(89+00)$ & $=$ & $\underline{\mathbf{1 1 9}}$ & $\mathbf{1 1 9}$ & \\
$(3-8)$ & $(36+0)+(84+00)$ & $=$ & $\underline{\mathbf{1 2 0}}$ & & 109 \\
$(3-7)$ & $(36+0)+(84-11)$ & $=$ & 109 & 120 \\
$(4-8)$ & $(36+1)+(84+00)$ & $=$ & $\underline{\mathbf{1 2 1}}$ & & \\
$(4-7)$ & $(36+1)+(84-11)$ & $=$ & 110 & & \\
\hline
\end{tabular}

The relationships are such that they show changes for the unit in the first, second and / or third position in the record of the number of atoms in the amino acids (in their side chains). At the same time, it is obvious that some sequences are generated from the series of natural numbers (two last columns). 
Appendix B. Two amino acid classes: AAs handled by class I and AAs handled by class II of enzymes aminoacyl-tRNA synthetases, respectively

Table B.1. The Combined Genetic Code Table (Rakočević, 2007a)

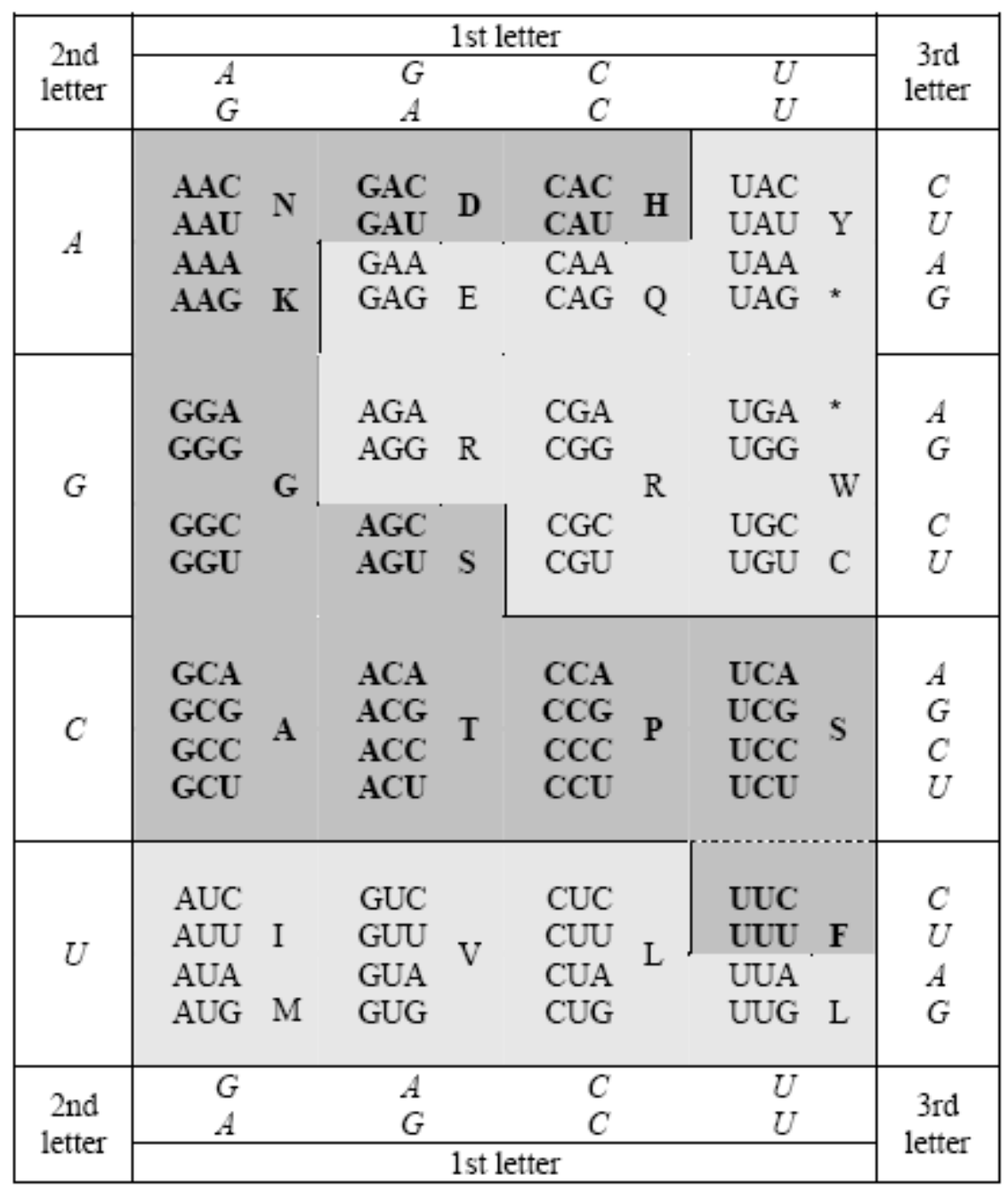

This new Table of the genetic code is realized in a specific combination of standard GCT and Swanson's Codon Path Cube (Swanson, 1984). In the combination are three different permutations from the total of 24. Bold positions (and dark tones): codons coding for AAs handled by class II of enzymes aminoacyltRNA synthetases (aaRS); non-bold positions: codons coding for AAs handled by class I of aaRS plus three "stop" codons, denoted with asterisk. As it is obvious, two groups of AAs are completely separated [Class I: (LVIM)+(YQERWC); Class II: (FSPTA)+(HDNKG)]. For the process of combining, the agreement-disagreement principle (Section 1.2) is valid as follows. In the standard GCT for all three positions in the codon (first, second and third letter), we have the maximal agreement - the same permutation (UCAG) all three times, but there is no agreement in the distinction into two classes in two separated areas (Wetzel, 1995). In the Codon Path cube the agreement in permutations is lower: for the first and second positions the UCGA and for the third position CUAG permutation; lower agreement in 
permutations, but greater in terms of separation into two classes of AAs, almost complete separation with one exception (Rakočević, 1997a). Finally, if we combine the GCT and Codon Path Cube, in the way shown here, we have a more lower agreement on permutations, but the greater agreement regarding the separation into two classes, they are completely separated. Apparently, this is also an analogy with the Heisenberg principle of uncertainty. [UCAG is used in the inner part of the Table for the first letter, and in the external part is UCGA permutation; for the third letter: in the inner part of the Table is UCGA, and in the external part CUAG permutation; UCGA permutation for both parts of the Table is valid for the second letter.] 
Appendix C. Two amino acid classes: AAs with odd and AAs with even number of hydrogen atoms

Table C.1. The Sukhodolets' Table of the number of hydrogen atoms

\begin{tabular}{|c|c|c|c|c|c|}
\hline \multirow{2}{*}{$\mathrm{n}$} & \multirow{2}{*}{ Amino acids } & \multicolumn{4}{|c|}{ Codon root } \\
\hline & & First & etter & Secor & letter \\
\hline 5 & Gly & G & & G & \\
\hline 7 & Ala Ser Asp cys & $\mathrm{G} \cup$ & G U & $\mathrm{C} \mathrm{C}$ & A G \\
\hline 8 & Asn & A & & A & \\
\hline 9 & Pro Thr Glu His & $\mathrm{C} A$ & G C & C $c$ & A A \\
\hline 10 & $G \ln$ & C & & A & \\
\hline 11 & Val Phe Met Tyr & $\mathrm{G} U$ & $\mathrm{~A} \cup$ & $\cup \cup$ & U A \\
\hline 12 & Trp & u & & G & \\
\hline $13-14$ & Leu Ile Arg Lys & $\mathrm{C} A$ & $C A$ & 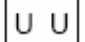 & $G A$ \\
\hline
\end{tabular}

The number of hydrogen atoms (n) within amino acid molecules, in relation to natural numbers series: 5 , (6), 7, 8, 9, 10, 11, 12, 13, 14 (Sukhodolets, 1985). First letter plus second letter equals a codon root (nucleotide doublet). The codon root plus third letter equals a complet codon. [Cf. this regularity of the hydrogen atoms within amino acids (in relation to natural number sequence) with the regularity of total number of atoms within nucleotides in standard GCT: within 64 codons (192 nucleotides) there are $2 \mathrm{x}$ 3456 atoms; 3456 in two inner as well as in two outer columns.]

Table C.2. The Sukhodolets' Table of the number of hydrogen atoms (Rakočević, 2011b)

\begin{tabular}{|c|c|c|c|}
\hline \multicolumn{4}{|c|}{ The number of $\mathrm{H}$ atoms (in brackets) and nucleons } \\
\hline \begin{tabular}{|l|l|}
$G(01) 01$ & $A(03) 15$
\end{tabular} & $|\mathrm{~S}(03) 31 \mathrm{D}(03) 59| \mathrm{C}(03) 47$ & (13) 153 & \multirow{4}{*}{$\begin{array}{c}(59 / 58) \\
569 / 686\end{array}$} \\
\hline $\begin{array}{ll}N(04) & 58 \\
\end{array}$ & $\mathrm{~T}(05) 45 \mathrm{E}(05) \quad 73 \mathrm{H}(05) 81$ & 298 & \\
\hline$Q(06) 72 \vee(07) 43$ & $\mathrm{~F}(07) 91 \mathrm{M}(07) 75 \mathrm{Y}(07) 107$ & 38 & \\
\hline$W(08) 130 R(10) 100$ & $\mathrm{~K}(10) 72$ I (09) $57 \mathrm{~L}(09) 57$ & (46) 416 & \\
\hline \multicolumn{3}{|c|}{$\begin{array}{l}569 \text { as neutron number and } 686 \text { as proton number! } \\
569-59=627-117 \\
686-58=628\end{array}$} & \\
\hline
\end{tabular}

Table C.2. The Sukhodolets' system of amino acids in a 4 x 5 arrangement. The Sukhodolets' Table, with a minimal modification (Sukhodolets, 1985): the system of 4 x 5 AAs. The shadow space: AAs with even number of hydrogen atoms $(4,6,8,10)$; the non-shadow space: AAs with odd number of hydrogen atoms $(1,3,7,9,11)$. In brackets: number of hydrogen atoms (within amino acid side chain) and out of brackets the number of nucleons. Nucleon number through a specific "simulation": 569 nucleons within two outer rows, as the number of neutrons, 
569, in all 20 AAs - within their side chains; and 686 nucleons within two inner rows, as the number of protons, 686, in all 20 AAs - within their side chains. Within 20 side chains of amino acid molecules there are 569 neutrons as well as 569 non-hydrogen protons. Within 20 side chains of amino acid molecules there are 117 hydrogen protons, what means 117 hydrogen atoms at the same time $(117=59+58)$.

Appendix D. "Project Details The constitution of group 3 of the periodic table" (IUPAC document)

"Project No.: 2015-039-2-200; Start Date: 18 December 2015; Division Name: Inorganic Chemistry Division; Division No.: 200

\section{Objective}

This project will deliver a recommendation in favor of the composition of group 3 of the periodic table as consisting either of

1. the elements Sc, Y, Lu and Lr, or

2. the elements Sc, Y, La and Ac.

The task group does not intend to recommend the use of a 32-column periodic table or an 18-column. This choice which is a matter of convention, rather than a scientific one, should be left to individual authors and educators. The task group will only concern itselve with the constitution of group 3 . Once this is established, one is free to represent the periodic table in an 18 or 32 column format.

\section{Description}

The question of precisely which elements should be placed in group 3 of the periodic table has been debated from time to time with apparently no resolution up to this point. This question has also received a recent impetus from several science news articles following an article in NaturêิA magazine in which the measurement of the ionization energy of the element lawrencium was reported for the first time.

We believe that this question is of considerable importance for chemists, physicists as well as students of the subject. Students and instructors are typically puzzled by the fact that published periodic tables show variation in the way that group 3 of the periodic table is displayed. The aim of the project is to assemble a task group to make a recommendation to IUPAC regarding the membership of group 3 of the periodic table.

Various forms of evidence have been put forward in support of each version of group 3. In the basis of this evidence arguments have been proposed while appealing to chemical as well as physical properties, spectral characteristics of the elements and criteria concerning the electronic configurations of their atoms. The task force will aim to evaluate all this evidence in order to reach a conclusion that encompasses these different approaches.

\section{Progress}

March 2016 - Project announcement published in Chem. Int. March 2016, p. 22;

http://dx.doi.org/10.1515/ci-2016-0213

Page last updated 19 March 2016" (Original text from IUPAC document) 


\section{REFERENCES}

Castro-Chavez, F. (2010) The rules of variation: amino acid exchange according to the rotating circular genetic code, J. Theor. Biol. 264, 711-721.

Castro-Chavez, F. (2011) The Quantum Workings of the Rotating 64-Grid Genetic Code, Neuroquantology 9 (4), 728-746.

Damjanović, Z., (1998) Logic core of genetic code, Glasnik Sect. Nat. Sci. Montenegrin Acad. Sci. Art (CANU) 12, 5-8.

Damjanović, Z.M., Rakočević, M. M. (2005) Genetic code: an alternative model of translation. Annals of New York Academy of Sciences, 1048, 517-523.

Damjanović, Z.M., Rakočević, M.M. (2006) Genetic Code: A new understanding of codon amino acid assignment, arXiv:q-bio/0611033v1 [q-bio.OT].

Dragovich, B., Dragovich, A. (2006) p-Adic Model of DNA Sequence and Genetic Code, arXiv:q-bio/0607018v1 [q-bio.GN].

Dragovich B., Dragovich A. (2010) p-Adic modeling of the genome and the genetic code. The Computer Journal; 53 (4): 432-441, 2010.

Dragovich, B. (2012) p-Adic structure of the genetic code, arXiv:1202.2353 [q-bio.OT].

Dragovich, B., Khrennikov A. Yu., Mišić N. Ž. (2017) Ultrametrics in the genetic code and the genome. Applied Mathematics and Computation 309, 350-358. arXiv:1704.04194 [qbio.OT].

Henderson, W., McIndoe, J.S. (2005) Mass Spectrometry of Inorganic, Coordination and Organometallic Compounds: Tools - Techniques - Tips, John Wiley \& Sons.

Kedrov, B.M. (1977) Predictions of Mendeleev in atomism - unknown Elements, Atomizdat, Moscow, [in Russian].

Knight, R.D., Freeland, S.J., Landweber, L.F. (2001) Rewiring the keyboard: evolvability of the genetic code, Nature reviews Genetics, Jan., 2001, Vol. 2, no 1, pp. 49-58.

Koruga, D.L. (1992) Neuromolecular Computing. Nanobiology, 1, 5-24.

Leunissen, J. A., De Jong, W.W. (1986) Phylogenetic trees constructed from hydrophobicity values of protein sequences. J. Theor. Biol. 119, 187-196.

Luchinskiy, G.P., Trifonov, D.N. (1981) Some problems of classification of chemical elements and the structure of periodic system of elements, in: The lerning of periodicity - the hystory and the contemporaryty, Edit. D.N. Trifonov, Nauka, Moskva (in Russian).

Maddox, J. (1992) New dimension for Mendeleev, Nature, 356, p. 13.

Maddox, J. (1994) The genetic code by numbers, Nature 367, p. 111. 
Mendelejeff, D.I. (1970) Zeitschrift für Chemie 12, 405-6., 1869. Reprinted in David M. Knight, ed., Classical Scientific Papers--Chemistry, Second Series, 1970; translation from German by Carmen Giunta.

Mišić, N.Ž. (2011) Nested numeric/geometric/arithmetic properties of shCherbak's prime quantum 037 as a base of (biological) coding/computing, Neuroquantology 9(4), 702-715.

Negadi, T. (2009) The genetic code degeneracy and the amino acids chemical composition are connected, Neuroquantology, Vol. 7, 1, 181-187; arXiv:0903.4131v1 [q-bio.OT].

Négadi, T. (2011) The Multiplet Structure of the Genetic Code, from One and Small Number, Neuroquantology 9(4), 767-771.

Negadi, T. (2014) The genetic code invariance: when Euler and Fibonacci meet, Symmetry: Culture and Science, Vol.25, No.3, 145-288, 2014; arXiv:1406.6092v1 [q-bio.OT].

Petoukhov, S. (2014) Matrix Genetics: Algebra of Projection Operators, Cyclic Groups and Inherited Ensembles of Biological Cycles, Proceedings of the 2nd International Conference "Theoretical Approaches to BioInformation Systems" (TABIS 2013), September 17 - 22, 2013 Belgrade.

Petoukhov, S. (2016) The system-resonance approach in modeling genetic structures. BioSystems 139, 1-11.

Popov, E.M. (1989) Strukturnaya organizaciya belkov (in Russian), Nauka, Moscow.

Qiu, Y., Zhu, L. (2000) The rearranged genetic code and its implications in evolution and biochemistry, Biosystems, Volume 56, Issues 2-3, May 2000, Pages 139-144.

Rakočević M.M. (1988) Three-dimensional model of the genetic code. Acta biologiae et madicinae experimentalis, 13, 109-116.

Rakočević, M.M. (1991) The coherence of the chemical and genetic code, Proceedings of Faculty of science (former: Faculty of phylosophy), Chemistry Section, 2, 1-29, Niš.

Rakočević, M. M. (1994) Logic of the Genetic Code, Naučna knjiga, Belgrade. (http://www.rakocevcode.rs)

Rakočević, M.M. (1997a) Two classes of the amino acyl-tRNA synthetases in correspondance with the Codon path cube, Bull. Math. Biol. 59, 645-648.

Rakočević, M.M. (1997b) Genetic code as a unique system, Studentski kulturni centar -Niš and Bina - Beograd, Serbia. (http://www.rakocevcode.rs)

Rakočević, M.M. (1998a) The harmony of periodic system of chemical elements, Flogiston, 7, 169-183 (in Serbian with an extended English summary).

Rakočević, M.M. (1998b) The genetic code as a Golden mean determined system, Biosystems, 46, 283-291.

Rakočević, M. M. (1998c) Whole-number relations between protein amino acids and their biosynthetic precursors, J. Theor. Biol. 191, 463 - 465 
Rakočević, M. M. (2000) The factors of the classification of protein amino acids, Glasnik of the Section of Natural Sciences of Montenegrin Academy of Sciences and art (CANU), 13, 273 294. (qbio.BM/0611004.)

Rakočević, M.M. (2004a) A harmonic structure of the genetic code, J. Theor. Biol. 229, 221234.

Rakočević, M.M. (2004b) Further Generalization of Golden Mean in Relation to Euler's "Divine" Equation, FME Transactions,32, (also in: arXiv:math/0611095 [math.GM])

Rakočević, M.M. (2006) Genetic Code as a Harmonic System, arXiv:q-bio/0610044 [q-bio.OT].

Rakočević, M.M. (2007a) Genetic Code as a Harmonic System: two Supplements, arXiv:qbio/0703011v2 [q-bio.OT], Table 3 in Supplement 2.

Rakočević, M.M. (2007b) A New Genetic Code Table, arXiv:q-bio/0703012 [q-bio.GN].

Rakočević, M.M. (2009) Genetic Code: The unity of the stereochemical determinism and pure chance, arXiv:0904.1161 [q-bio.BM].

Rakočević, M.M. (2011a) Genetic Code: Four Diversity Types of Protein Amino Acids, arXiv:1107.1998v2 [q-bio.OT].

Rakočević, M.M. (2011b) Genetic code as a coherent system. Neuroquantology 9 (4), 821-841;

Rakočević, M. M. (2014) Golden and harmonic mean in the genetic code, Proceedings of the 2nd International Conference "Theoretical Approaches to Bioinformation Systems" (TABIS 2013), September 17 - 22, 2013 Belgrade.

Rakočević, M.M., Jokić, A. (1996) Four stereochemical types of protein amino acids: synchronic determination with chemical characteristics, atom and nucleon number. J. Theor. Biol. 183, 345-349.

Sacks, Lawrence (2006) Concerning the position of hydrogen in the periodic table, Foundations of Chemistry, 2006, 8, pp. 31-35.

Shcherbak, V. I. (1993) Twenty canonical amino acids of the genetic code: the arithmetical regularities. Part I. J Theor. Biol. 162, 399-401.

Shcherbak, V.I. (1994) Sixty-four triplets and 20 canonical amino acids of the genetic code: the arithmetical regularities. Part II, J. Theor. Biol., 166, 475-477.

Shcherbak, V. I. (2003) Arithmetic inside the universal genetic code, Biosystems 70, 187-209.

Shcherbak, V.I. (2008) The arithmetical origin of the genetic code, in: The Codes of Life, Springer, Berlin.

Schonberger, Martin (1980) The I Ching and Genetic Code, ASI, New York.

Spinadel, V.W. de (1998) The Metallic Means and design. In NEXUS II- Architecture and Mathematics. Ed.: Kim Williams, 1998.

Spinadel, V.W. de (1999) The family of metallic means, Visual Mathematics, 1 (3), 1-16, http//members.tripod.com/vismath1/spinadel/.

Stakhov, A. P. (1989) The Golden section in measurem. theory, Computers Math. Applic., 17, 613-638. 
Stent, G.S. (1969) The Coming of the Golden Age, Freeman, New York.

Stewart, Philip (2010). Charles Janet: unrecognized genius of the Periodic System, Foundations of Chemistry. 12, 5-15.

Sukhodolec, V.V. (1985) The meaning of the genetic code: the reconstruction of the stages of prebiological evolution (in Russian), Генетика, XXI, 10, 1589 - 1599.]

Van Nostrand's Scientific Encyclopedia $(1983) 6^{\text {th }}$ Ed, New York.

Verkhovod, A.B. (1994) Alphanumerical Divisions of the Universal Genetic Code: New Divisions Reveal New Balances. J. Theor. Biol. 170, 327-330.

Weaver, R.F. (2012) Molecular Biology, fifth edition, McGraw-Hill, New York.

Wetzel, R. (1995) Evolution of the Aminoacyl-tRNA Synthetases and the Origin of the Genetic Code. J. Mol. Evol. 40, 545-550.

Wohlin, Åsa (2015) Numeral series hidden in the distribution of atomic mass of amino acids to codon domains in the genetic code, J. Theor. Biol., 369, 95-109.

Yang, C. M. (2004) On the 28-Gon Symmetry Inherent in the Genetic Code Intertwined with Aminoacyl-tRNA Synthetases-The Lucas Series, Bulletin of Mathematical Biology, 66, $1241-1257$. 